Cahiers
de a $\begin{gathered}\text { Recherche } \\ \text { sur les Droits }\end{gathered}$

Cahiers de la recherche sur les droits fondamentaux

16 | 2018

Les partis politiques

\title{
Le statut partisan du chef de l'État turc
}

The Partisan Status of the Turkish Head of State

\section{Aysegul Fistikci}

\section{CpenEdition}

Journals

Édition électronique

URL : https://journals.openedition.org/crdf/313

DOI : $10.4000 /$ crdf.313

ISSN : 2264-1246

Éditeur

Presses universitaires de Caen

Édition imprimée

Date de publication : 16 novembre 2018

Pagination : 79-99

ISBN : 978-2-84133-901-3

ISSN : 1634-8842

Référence électronique

Aysegul Fistikci, «Le statut partisan du chef de l'État turc », Cahiers de la recherche sur les droits

fondamentaux [En ligne], 16 | 2018, mis en ligne le 16 novembre 2019, consulté le 15 novembre 2022.

URL : http://journals.openedition.org/crdf/313 ; DOI : https://doi.org/10.4000/crdf.313 


\title{
Le statut partisan du chef de l'État turc
}

\author{
Aysegul FISTIKCI \\ Doctorante en droit public à l'université de Caen Normandie \\ Centre de recherche sur les droits fondamentaux et les évolutions du droit (CRDFED, EA 2132)
}

I. Le statut partisan du chef de l'État: une situation de fait avant la réforme de 2017

A. Un statut partisan par intermittence avant la réforme de l'élection au suffrage universel direct

1. Le caractère limité de l'exigence de neutralité imposée par le statut constitutionnel

2. L'attractivité du statut présidentiel pour les partis politiques et les différents « usages » du statut partisan du chef de l'État

B. L'intervention de l'élection directe: la systématisation du chef de l'État partisan

1. La cause de la réforme: une réforme par le parti majoritaire contre les autorités de tutelle

2. L'effet de la réforme: une réforme pour le parti majoritaire et son leader

II. La constitutionnalisation du chef de l'État partisan par la révision de 2017

A. La pérennisation des acquis de la présidentialisation du régime

1. Un confusionnisme des pouvoirs législatif et exécutif entretenu par la présidence partisane

2. La duplicité de la fonction présidentielle

B. L'impossible retour à la neutralité présidentielle?

1. L'accentuation de la logique partisane de l'élection présidentielle

2. Le développement de la démocratie intra-partisane: un correctif envisageable?

\section{Annexes}

Présentation succincte des partis politiques cités dans l'article

Principaux repères chronologiques

Selon l'article 68, alinéa 2 de la Constitution turque du 7 novembre $1982^{1}$, «les partis politiques sont les éléments indispensables de la vie politique démocratique $»^{2}$. Mais cette disposition n'est pas tant révélatrice de la philosophie qui imprègne la Constitution de la III ${ }^{e}$ République turque lors de son adoption à la suite du coup d'État militaire du 12 septembre $1980^{3}$. Ainsi, selon Serap Yazıc1, la philosophie de cette Constitution fait état d'une double méfiance à l'égard du peuple et de ses représentants. Le constituant de 1982 met donc en place un certain nombre d'autorités

1. Resmî Gazete, 9 novembre 1982, 17863, 1. Mükerrer, p. 17.

2. Les traductions du turc vers le français sont, sauf mention contraire, effectuées par nos soins.

3. Il a fallu attendre le référendum de 1987 pour que les partis politiques et leurs leaders ne redeviennent officiellement les acteurs de la vie politique à la suite de la dissolution des partis politiques par le pouvoir militaire en place par la loi no 2533 du 16 octobre 1981 (Resmî Gazete, 16 octobre 1981 , 17486 Mükerrer). 
de tutelle chargées de veiller, voire de contenir, le pouvoir démocratique ${ }^{4}$.

Parmi ces autorités se trouve la présidence de la République chargée de surveiller les institutions représentatives menées par les partis politiques. Le constituant lui attribue pour cela des prérogatives conséquentes, renforçant ainsi l'exécutif dans son aile présidentielle. Afin d'assumer un tel rôle, la principale caractéristique du président repose sur sa neutralité, conformément à l'acception du chef de l'État en régime parlementaires.

Cette neutralité présidentielle ne s'est pas imposée avec évidence dans les régimes républicains turcs depuis 1920. La I ${ }^{\text {re }}$ République turque a connu le régime à parti unique dont le leader a été le président. Ce n'est que par le passage au multipartisme en 1946 que la question de la neutralité présidentielle s'est posée, notamment par le parti d'opposition, à laquelle le président İsmet Inönü a répondu par une déclaration en date du 12 juillet 1947 en s'engageant à se tenir à égale distance du parti majoritaire et de celui de l'opposition ${ }^{6}$. Mais il a fallu attendre la Constitution de 1961, adoptée à la suite du coup d'État militaire du 27 mai 1960, pour une véritable consécration de la neutralité présidentielle dans la lettre de la Constitution qui prenait toutefois, en pratique, la forme d'un contrôle de l'armée sur la présidence de la République.

Le régime politique turc actuel consacre initialement un régime parlementaire rationalisé avec un renforcement du statut présidentiel. Toutefois, les réformes constitutionnelles successives ont fortement impacté cette qualification, et notamment celles touchant au statut présidentiel de 2007 prévoyant l'élection présidentielle au suffrage universel direct, et celle de 2017 mettant en œuvre un régime «de gouvernement de la présidence de la République » dans lequel on retrouve un exécutif monocéphale constitué par le seul président de la République et qui n'est plus soumis à l'obligation d'impartialité vis-à-vis des partis politiques comme c'est classiquement le cas en régime parlementaire.

Se pose donc ici la question de l'influence du statut présidentiel et celle de son impact sur la qualification du régime politique. Il s'agit plus spécifiquement de comprendre quelles sont les implications d'un statut partisan du chef de l'État sur l'équilibre des pouvoirs dans un régime initialement conçu comme parlementaire.

Contrairement à ce qui semble perceptible, le statut partisan du chef de l'État a été amorcé plus tôt dans la pratique du régime politique de la $\mathrm{III}^{\mathrm{e}}$ République turque, avant même l'élection du président au suffrage universel direct. La nature partisane du statut du chef de l'État en Turquie, bien qu'impropre au régime parlementaire, constitue un élément essentiel de la qualification du régime politique, notamment en ce qu'il aboutit à une forme de concentration, voire, dans certains contextes, de confusion des pouvoirs.

Après avoir constaté que le statut partisan du chef de l'État est ainsi une réalité connue du régime politique turc, quoique de manière aléatoire avant la révision constitutionnelle adoptée par le référendum du 16 avril 2017 (I), nous verrons que cette dernière a finalement constitutionnalisé le statut partisan du chef de l'État en rendant quasiment impossible tout retour à la neutralité présidentielle pourtant nécessaire à l'équilibre du régime (II).

\section{Le statut partisan du chef de l'État: une situation de fait avant la réforme de 2017}

Malgré une conception neutre de la présidence de la République $^{7}$, la pratique du régime politique turc connaît des présidences partisanes depuis ses débuts, et ce bien avant la révision constitutionnelle de 2007 visant à désigner le président au suffrage universel direct (A). Cette dernière a toutefois eu pour effet de systématiser ce statut partisan en plaçant officiellement les partis au cœur du processus électoral présidentiel et du fonctionnement du régime (B).

\section{A. Un statut partisan par intermittence avant la réforme de l'élection au suffrage universel direct}

Le mode de désignation du président constitue un véritable enjeu dans la mise en œuvre de sa neutralité. Or, les élections présidentielles par la Grande Assemblée nationale de Turquie (GANT) ne se sont pas toujours déroulées conformément à l'esprit de la Constitution, qui tend, dans la lignée de la précédente Constitution de 1961, à inhiber l'emprise des factions partisanes sur cette désignation et à privilégier le consensus. La pratique politique a ainsi permis la désignation à la présidence de la République de certains leaders de la majorité du fait des mécanismes constitutionnels pas toujours en adéquation avec l'exigence de neutralité (1), ce qui est dû à une certaine culture politique faisant du chef de l'État un leader partisan en opposition à la norme constitutionnelle (2).

4. Voir S. Yazıcı, «1982 anayasası ve cumhurbaşkanlığı», in Prof. Dr. Erdal Onar’a Armağan, Cilt-I, Ankara, Ankara Üniversitesi yayınları, 2013, p. 231.

5. Voir B. Constant, Cours de politique constitutionnelle, Genève - Paris, Slatkine, 1982, t. I, p. 178.

6. Voir M. Erdoğan, Türkiye'de Anayasalar ve Siyaset, $8^{\mathrm{e}}$ éd., Ankara, Liberte Yayınları, 2012, p. 101.

7. La version initiale de l'article 101 de la Constitution telle qu'adoptée par le référendum du 7 novembre 1982 disposait ainsi: «Le président de la République est élu par la Grande Assemblée nationale de Turquie parmi ses membres ayant quarante ans révolus et un enseignement universitaire ou parmi les citoyens turcs présentant ces caractéristiques et remplissant les conditions d'éligibilité des députés pour une durée de sept ans. / La présentation des candidats à la présidence de la République des personnes extérieures à la Grande Assemblée nationale de Turquie n’est possible que sur proposition écrite d'au moins un cinquième du nombre total des membres de l'Assemblée. / Nul ne peut être élu deux fois président de la République. / La personne élue président de la République voit, le cas échéant, la rupture de ses liens avec son parti et l'achèvement de sa qualité de membre de la Grande Assemblée nationale de Turquie» (Resmî Gazete, 9 novembre 1982, 17863, 1. Mükerrer, p. 26). 


\section{Le caractère limité de l'exigence de neutralité imposée par le statut constitutionnel}

La Constitution de 1982 consacre un régime parlementaire ${ }^{8}$ avec un renforcement de l'exécutif, et plus spécifiquement de la présidence de la République?. Le président est envisagé comme un arbitre entre les pouvoirs ${ }^{10}$ et détient des prérogatives conséquentes afin d'assumer un tel rôle ${ }^{11}$, conjugué à une irresponsabilité politique ${ }^{12}$ impliquant sa neutralité ${ }^{13}$. Il s'agit ici d'une obligation constitutionnellement consacrée: l'article 101, alinéa 4 de la version originelle de la Constitution de 1982 imposait, le cas échéant, la rupture des liens entre la personne élue à la présidence et son parti d'origine ${ }^{14}$.

Afin de préserver le caractère neutre du statut présidentiel, les constituants de 1982 ont maintenu les dispositions mises en place lors de la précédente Constitution de 1961 dont l'objectif était d'écarter la mainmise d'un parti ou d'un camp politique donné sur l'élection présidentielle en favorisant le consensus autour de cet organe clé. Le maintien de la condition de majorité requise pour l'élection présidentielle paraît alors tout indiqué. En effet, s'agissant d'une élection à une majorité qualifiée des deux tiers de la GANT ${ }^{15}$, l'objectif est d'encourager le consensus entre les différents partis la composant. Mais, dans le même temps, le but est aussi d'écarter, tant que possible, les tractations partisanes autour de cette élection afin de favoriser son impartialité vis-à-vis des partis politiques. En prescrivant ainsi l'élection à bulletin secret dans la même disposition, le constituant s'adresse davantage à l'aptitude du parlementaire d'élire le président le mieux à même d'exercer cette fonction, qu'aux partis politiques enclins à soumettre la désignation de cet organe neutre à la discipline de parti fortement ancrée dans la culture politique du pays.

Toutefois, parvenir à un tel consensus n'est pas chose aisée, notamment du fait de la polarisation des camps politiques. C'est ce qu'a démontré l'expérience de la II ${ }^{e}$ République turque durant laquelle, à la suite de la présidence de Fahri Korutürk ${ }^{16}$, aucun président n'a pu être élu par la GANT en six mois ${ }^{17}$. Cette crise a constitué l'une des justifications du coup d'État du 12 septembre $1980^{18}$. En effet, la recherche d'une telle majorité afin de favoriser le consensus est logique, mais encore faut-il s'assurer que la culture politique des électeurs, en l'occurrence les parlementaires, s'inscrive dans une telle démarche. Or, si l'élection du président présente des enjeux politiques considérables pour les factions politiques, elle se transformera immanquablement en un terrain d'affrontement inter-partisan supplémentaire. D'autant que, sur ce terrain-ci, l'opposition détient une faculté d'empêcher qui peut potentiellement s'exercer comme un pouvoir de nuisance, et non comme un élément constructif permettant d'aboutir à une élection consensuelle de la présidence de la République. C'est pourquoi les constituants de 1982 ont rationalisé la procédure de l'élection présidentielle afin d'éviter ce type de crise politique pouvant mener à la paralysie institutionnelle ${ }^{19}$. La Constitution de 1982 prévoit donc une limitation du nombre de tours de l'élection présidentielle à quatre, ainsi qu'une limitation temporelle

8. En effet, il existe des moyens d'action entre les pouvoirs, et notamment la classique responsabilité gouvernementale (article 112 de la Constitution de 1982, Resmî Gazete, 9 novembre 1982, 17863, 1. Mükerrer, p. 30).

9. Voir E. Özbudun, Türk Anayasa Hukuku, $15^{\mathrm{e}}$ éd., Istanbul, Yetkin Yayınlar1, 2014, p. 64.

10. L'article 104 de la Constitution de 1982 précisant la fonction et les prérogatives présidentielles (qui reste en vigueur) dispose comme suit : «Le président de la République est le chef de l'État. À ce titre, il représente l'unité de la République de Turquie et de la nation turque; il veille à l'application de la Constitution et au fonctionnement ordonné et harmonieux des organes de l'État» (Resmî Gazete, 9 novembre 1982, 17863, 1. Mükerrer, p. 27).

11. La suite de l'article 104 prévoit une longue liste de prérogatives du chef de l'État qui touchent aux différentes fonctions étatiques que sont les fonctions exécutive, législative et juridictionnelle. Les prérogatives démontrant un véritable renforcement de la présidence sont ses pouvoirs de nomination qui sont des pouvoirs propres et qui ne peuvent être contrôlés par aucune juridiction. Il nomme le Premier ministre et accepte sa démission et, sur proposition de celui-ci, il nomme et révoque les ministres. Le président a également des prérogatives élargies en périodes exceptionnelles comme l'état d'urgence, il dispose de nombreuses prérogatives liées à son rôle de gardien de la Constitution telles que le renvoi des lois en seconde lecture, la saisine de la Cour constitutionnelle, le choix de soumettre la loi de révision constitutionnelle au référendum, etc. En outre, il participe à l'édiction des décrets-lois qui doivent être signées de sa part. Toutefois, le chef de l'État ne disposait pas, avant 2017, d'un pouvoir de dissolution à sa discrétion, celui-ci étant encadré par un certain nombre de conditions (article 116 dans sa version antérieure à la révision de 2017; voir infra). L'article 105 dispose que le président peut accomplir des actes seul, sans contreseing, et qui ne peuvent faire l'objet d'aucun recours, avec un renvoi aux dispositions constitutionnelles et législatives, sans précision sur le contenu exact de ces actes.

12. Article 105 de la Constitution de 1982 (Resmî Gazete, 9 novembre 1982, 17863, 1. Mükerrer, p. 29).

13. En effet, lors de sa présentation de la Constitution, le général Evren justifiait le choix du renforcement de la présidence plutôt que celle du gouvernement par sa neutralité partisane: «Certaines prérogatives ont été attribuées au gouvernement qui est partial. Cependant, les prérogatives pouvant donner lieu à de sérieux affrontements entre majorité et opposition ont été attribuées au président. En dehors de cela, il y a des prérogatives qui doivent être conférées au président, qu'il ne serait ni correct ni possible, par essence, de les attribuer à un autre organe» (cité par E. Özbudun, Türk Anayasa Hukuku, p. 64).

14. Jusqu'à la révision constitutionnelle de 2017 (voir infra).

15. Article 102, alinéa $1^{\text {er }}$ de la Constitution de 1982 (Resmî Gazete, 9 novembre 1982, 17863, 1. Mükerrer, p. 27).

16. Mandat présidentiel du 6 avril 1973 au 6 avril 1980 (pour la liste des mandats présidentiels, voir le site officiel de la présidence de la République de Turquie: https://www.tccb.gov.tr/cumhurbaskanlarimiz).

17. Voir Ș. Özsoy, "Cumhuriyet'in kurulușundan bu yana Türkiye'de cumhurbașkanlığı seçimleri meselesi», in Prof. Dr. Erdal Onar'a Armağan, Cilt-I, p. 396; K. H. Yavuz, Türkiye'de siyasal sistem arayışı ve yürütmenin güçlendirilmesi, Ankara, Seçkin, 2000, p. $400-402$.

18. K. Halûk Yavuz l'évoque en ces termes: "C’est notamment le blocage de l'élection qui a créé de sérieuses difficultés et a constitué l'un des motifs les plus importants de l'intervention» (K. H. Yavuz, Türkiye'de siyasal sistem arayışı ve yürütmenin güçlendirilmesi, p. 423). L'autre étant l'incapacité des autorités civiles de lutter contre le terrorisme (R. Akın, Gazi'den günümüze cumhurbaşkanlı̆gl, 1923-20o7, Istanbul, Türkiye is bankası Kültür Yayınları, 2009, p. 117).

19. Ş. Özsoy, "Cumhuriyet'in kuruluşundan... », p. 407. 
de la procédure de l'élection fixée à trente jours. La majorité requise est abaissée à la majorité absolue à partir du troisième tour en continuité avec les dispositions de 1961 mais seuls les deux candidats ayant reçu le plus de suffrages au tour précédent peuvent participer au quatrième. Le candidat élu devra obtenir la majorité absolue du nombre total des députés ${ }^{20}$. Le constituant entend ainsi concilier la recherche de l'élection consensuelle avec les éléments de rationalisation d'un tel processus, en y ajoutant un mécanisme particulièrement dissuasif à l'encontre des électeurs qui est le renouvellement systématique de la GANT en cas d'échec à élire le président ${ }^{21}$.

Cependant, ces mêmes dispositions, en ce qu'elles rationalisent l'élection présidentielle, créent aussi les failles d'un système que les partis politiques ont pu exploiter en élisant des chefs de majorité à la présidence de la République, mettant ainsi à mal ce fragile équilibre conçu par les constituants. Alors qu'il s'agit d'un objectif constitutionnel, l'élection n'est pas consensuelle dans les faits car la rationalisation du processus aboutit à ce qu'un parti politique majoritaire n'est pas contraint à rechercher le consensus puisqu'il lui suffit de patienter jusqu'aux troisième et quatrième tours pour élire le candidat soutenu par le parti ${ }^{22}$. La rationalisation aurait dû prévoir une telle contrainte en imposant la recherche du consensus qui ne s'inscrit pas dans la culture politique des parlementaires car ce n'est que lorsque celle-ci est imposée par le contexte politique qu'elle a effectivement $\operatorname{lieu}^{23}$. La rationalisation ainsi conçue en 1982 donne finalement la capacité de désigner au parti majoritaire seul le président de la République ${ }^{24}$. C'est ainsi que, dans les faits, les partis détenant la majorité au sein de la GANT ne cherchaient pas à désigner une personnalité apte à fédérer plusieurs camps politiques. Cela a donné généralement lieu à l'élection des anciens Premiers ministres et chefs des majorités tels que Turgut Özal en 1989 et Süleyman Demirel en 1993 (par la coalition majoritaire), ainsi qu'à l'élection de la deuxième tête du parti majoritaire de l'AKP, Abdullah Gül en 2007. Toutes ces figures politiques, aussi expérimentées politiquement qu'elles soient en tant que leaders politiques de premier plan, n'ont pas les caractéristiques d'une personnalité pouvant incarner cette neutralité. Seule l'élection d'A. Necdet Sezer a connu une négociation d'une assez grande ampleur par un accord entre les chefs de parti de la coalition majoritaire (ANAP, DSP, MHP ${ }^{25}$ ) et l'opposition (FP, DYP) sous l'impulsion du Premier ministre de l'époque, Bülent Ecevit ${ }^{26}$.

La volonté de rationaliser la procédure électorale présidentielle ne constituait pas une limite insurmontable. Toutefois, le système électoral législatif turc ${ }^{27}$ a été la principale cause de l'échec de l'application d'un tel processus, notamment en ce qu'il institue, encore aujourd'hui, un barrage électoral à l'échelle nationale fixé à $10 \%{ }^{28}$, ayant pour effet d'empêcher les partis politiques ayant obtenu un score en deçà d'obtenir le moindre siège. Alors que la représentation d'une pluralité de partis politiques favoriserait davantage la recherche du consensus, l'instauration d'un tel barrage, sous couvert de stabilité politique, permet le plus souvent d'avantager la représentation de certains partis politiques qui concentrent une force politique conséquente au sein du Parlement et peuvent ainsi élire seuls le président.

Ce ne sont pas seulement les dispositions relatives à la procédure électorale qui impactent la neutralité présidentielle. Les dispositions fixant les conditions d'éligibilité du président à l'article 101 de la Constitution permettent dans l'esprit des constituants d'asseoir le pouvoir neutre présidentiel en désignant une personnalité présentant les aptitudes nécessaires à l'exercice d'un tel pouvoir. Parmi celles-ci se trouve la condition de parrainage spécifique aux candidats non parlementaires imposant le parrainage d'un cinquième des parlementaires. Se pose alors la question de l'efficacité d'une telle disposition concernant la neutralité présidentielle car celle-ci favorise les candidatures des parlementaires ayant potentiellement une appartenance partisane plus ancrée que des candidatures extraparlementaires.

Le constituant a également tenté de favoriser la neutralité présidentielle en dissociant son mandat du mandat parlementaire mais aussi, et surtout, en prévoyant le caractère non renouvelable du mandat présidentiel ${ }^{29}$. Là encore, il s'agit d'un héritage de la II ${ }^{\mathrm{e}}$ République construite en opposition à la Ire qui prévoyait un mandat présidentiel

20. L'Assemblée consultative avait alors préconisé une majorité simple pour le quatrième tour, ce qu'a refusé le Conseil de sécurité nationale du fait des critiques émises lors de l'élaboration constitutionnelle et tenant à ce que le président ne serait dans ce cas que l'élu d'un parti (Ş. Özsoy, "Cumhuriyet'in kuruluşundan...", p. 406).

21. Article 102, alinéa 3 de la Constitution de 1982

22. Voir B. Tanör, N. Yüzbaşığlu, 1982 Anayasasına göre türk anayasa hukuku, 12 éd., Istanbul, Beta, 2012, p. 321

23. Les élections de Süleyman Demirel (mandat du 16 mai 1993 au 16 mai 200o) et d'A. Necdet Sezer (mandat du 16 mai 2000 au 28 août 2007 ) ont pu aboutir par le biais d'une entente de plusieurs partis politiques.

24. Voir H. Özdemir, Devlet krizi. T.C. cumhurbaşkanlı̆̆ı seçimleri, Istanbul, Afa Yayınları, 1989, p. 37.

25. Voir en annexe la présentation des partis politiques turcs cités dans l'article.

26. Mais, là encore, l'ancien président de la Cour constitutionnelle qu'était alors A. Necdet Sezer ne fut élu qu'au troisième tour du fait de la présence de candidats frondeurs représentant leur affiliation politique (R. Akın, Gazi'den günümüze cumhurbaşkanlığı, 1923-2007, p. 174).

27. Mode de scrutin proportionnel faisant application du système D’Hondt quant à la répartition des restes conformément à l'article 2 de la loi $\mathrm{n}^{\circ} 2839$ du 10 juin 1983 sur l'élection des députés (Resmî Gazete, 13 juin 1983, 18076, p. 8).

28. Article 33, alinéa $1^{\text {er }}$ de la loi $\mathrm{n}^{\circ} 2839$ du 10 juin 1983 sur l'élection des députés modifié par l'article 9 de la loi nº 3377 du 23 mai 1987 (Resmî Gazete, 3 juin 1987, 19476, p. 3), et par l'article 20 de la loi nº 7102 du 13 mars 2018 (Resmî Gazete, 16 mars 2018, 30362).

29. L'article 101 de la Constitution de 1982 prévoyait initialement un septennat non renouvelable pour le président tandis que l'article 77 mettait en place un quinquennat pour la durée de la législature. 
calqué sur le mandat législatif ${ }^{3 \circ}$, aboutissant à l'élection du leader du parti unique jusqu'en 1945, puis du leader de la majorité en place ${ }^{31}$, conjuguée à un renouvellement conséquent de leur mandat ${ }^{32}$. C'est la raison pour laquelle le découplage des mandats présidentiel et législatif et la limitation à un mandat unique ${ }^{33}$ se sont imposés au constituant de 1961 puis à celui de 1982. Cette limitation apparaît comme une condition essentielle de la neutralité présidentielle, évitant ainsi la présence d'un président candidat à sa propre succession susceptible de négocier sa réélection avec les électeurs qui sont constitués principalement par les partis politiques. Toutefois, cette disposition n'a pas toujours été efficace dans la mesure où la discordance des mandats a conduit Turgut Özal à présenter sa candidature à l'élection présidentielle afin de maintenir un poids dans le fonctionnement du régime lorsqu'il a senti que son parti, l'ANAP, n'allait plus disposer de la majorité ${ }^{34}$.

Ainsi, l'inefficacité de ces dispositions est à souligner car elles n'ont abouti que très rarement à une élection consensuelle. En effet, le renforcement du statut présidentiel fait de cet organe un pouvoir particulièrement attractif pour les organisations partisanes et notamment celles qui disposaient d'une majorité confortable afin d'élire à elles seules le leader de leur majorité.

\section{L'attractivité du statut présidentiel pour les partis politiques et les différents " usages " du statut partisan du chef de l'État}

Les pratiques politiques concernant les liens entre le président et son parti ont été variables dans l'histoire républicaine turque. Alors que celles provenant de la première Constitution républicaine de 1924 tendent vers l'élection du leader majoritaire à la présidence de la République, la deuxième a entendu instituer une présidence comme véritable autorité de tutelle mais sous le contrôle de l'armée. Ainsi, toutes les personnalités élues lors de cette $\mathrm{II}^{\mathrm{e}}$ République étaient issues de cette institution et n'étaient pas rattachées à un parti politique ${ }^{35}$ contrairement à la pratique observée sous la Constitution de 1982, faisant état de l'élection par intermittence de présidents ayant une appartenance partisane certaine. La différence de cette pratique s'explique d'abord par un moindre interventionnisme de l'armée dans ce processus ${ }^{36}$, mais cela a aussi un lien avec le choix opéré par les constituants de 1982 consistant à renforcer la présidence de la République en lui attribuant un rôle clé dans le fonctionnement des institutions. En effet, l'institution présidentielle n'a pas été conçue comme partisane lors de l'élaboration de la Constitution. À ce moment précis, le fait que, dans l'histoire de la République turque, tous les présidents élus aient été issus de l'armée, exception faite de Celal Bayar, est particulièrement révélateur d'une certaine conception de la présidence et de sa neutralité. C'est donc en concevant l'institution présidentielle comme une autorité de tutelle, qui était censée être la chasse gardée de l'armée, que les rédacteurs de la Constitution de 1982 ont renforcé cette institution. Bien que le président ait été alors envisagé comme une autorité régulatrice, l'importance de ses prérogatives conjuguée à une responsabilité limitée est propice à transformer cette institution en terrain d'affrontements politiques inter-partisans malgré sa neutralité constitutionnelle.

Lorsqu'un parti a les moyens d'élire le président, seul ou en coalition, l'interrogation porte sur le choix de la personnalité à installer à la présidence. La logique parlementaire du régime initialement consacrée tendrait plutôt à encourager la pratique d'asseoir ces leaders, non dans le fauteuil du président, qui, malgré son positionnement renforcé, ne gouverne pas selon les dispositions constitutionnelles, mais bien dans celui du Premier ministre, qui conduit la politique générale déterminée avec son gouvernement ${ }^{37}$. Cependant, la présidence de la République révèle une position stratégique dans le fonctionnement des institutions, et la nature du statut présidentiel constitue un des éléments définissant le régime politique dans lequel il s'inscrit. Ainsi, le parti politique qui souhaite gouverner sans entraves a tout intérêt à cibler la présidence de la République, la conjugaison d'une majorité et d'un président de même bord limitant considérablement les obstacles à la mise en œuvre de la politique du parti au pouvoir. D'un point de vue de stratégie politique, il apparaît également que l'élection présidentielle d'une personnalité gardant officieusement une appartenance partisane permet à son parti de maintenir un poids politique considérable malgré un éventuel changement de majorité. C'est ce que démontre l'élection de Turgut Özal, membre fondateur

30. Avec un président élu à la majorité simple des votants à chaque renouvellement de l'Assemblée et dont la durée du mandat était identique à celle de la législature selon l'article 31 de la Constitution de 1924 (Resmî Gazete, 24 avril 1924, 71, p. 576 sq.; pour la version en turc moderne, voir Resmî Gazete, 15 janvier 1945, 5905).

31. M. Kemal Atatürk (du 29 octobre 1923 au 10 novembre 1938), İsmet İnönü (du 11 novembre 1938 au 22 mai 1950) et Celal Bayar (du 22 mai 1950 au 27 mai 1960).

32. Trois mandats pour M. Kemal Atatürk et İsmet Inönü, et deux mandats pour Celal Bayar dont le second a été interrompu par le coup d'État du 27 mai 1960.

33. Jusqu'à la révision constitutionnelle de 2007 .

34. Cela a été beaucoup critiqué par Süleyman Demirel pour lequel le changement de majorité en 1991, avec la défaite de l'ANAP, a ôté toute légitimité au président qui aurait dû démissionner (Z. Çaglıyan Içener, «Türkiye’de baskanlık sistemi tartısmalarının yakın tarihi: Özal ve Demirel’in siyasi mülahazalar1", Bilig, $\mathrm{n}^{\circ} 75$, automne 2015, p. 319).

35. Cemal Gürsel (du 27 mai 1960 au 28 mars 1966), Cevdet Sunay (du 28 mars 1966 au 28 mars 1973) et Fahri Korutürk (du 6 avril 1973 au 6 avril 1980 ).

36. Même si les liens entre l'armée et la politique n'ont pas disparu (voir infra).

37. Article 112, alinéa ${ }^{\text {er }}$ de la Constitution de 1982: «Le Premier ministre, en sa qualité de président du Conseil des ministres, assure la coopération entre les ministères et veille à l'exécution de la politique générale du gouvernement. Le Conseil des ministres est solidairement responsable de l'exécution de cette politique» (Resmî Gazete, 9 novembre 1982, 17863, 1. Mükerrer, p. 30). 
de l'ANAP, parti ayant pu construire son influence du fait notamment de l'interdiction des anciens partis politiques à la suite du coup d'État de 1980 et l'adoption de la loi relative à la dissolution des partis politiques ${ }^{38}$. C'est ce qui explique le succès d'Özal dans les années 1980 puisqu'il devient d'abord Premier ministre en 1983 avec une majorité confortable à la GANT ${ }^{39}$, fonction à laquelle il se maintient à la suite des élections de 1987 avec une majorité plus importante malgré une baisse en pourcentage de voix ${ }^{40}$. Cette baisse significative, due notamment à la reprise d'activité des anciens partis politiques, a été le signe d'un essoufflement pour le parti, ce qui a conduit Özal à briguer la présidence de la République, poste qu'il occupera à partir du 9 novembre $1989^{41}$. Mais la candidature d'Özal à la présidence a été très contestée, depuis l'opposition jusqu'à ses rangs, puisqu'il n'incarnait en rien la neutralité caractéristique du statut présidentiel et son mandat en fut la démonstration. Sa présidence peut être distinguée en deux périodes: la première étant celle durant laquelle son parti était majoritaire à la GANT, jusqu'aux élections de 1991, puis la seconde avec la perte de la majorité de l'ANAP au profit du DYP de Demirel, qui a pu former une coalition avec le SHP d'Erdal Inönü, avatar du CHP, jusqu'à la fin du mandat d'Özal suite à son décès en $1993^{42}$. Alors que le président Özal détenait une influence considérable sur son parti durant la première période ${ }^{43}$, ce qui a donné lieu à une forme de présidentialisation du régime politique sous la dénonciation par ses opposants politiques de violation de la Constitution du fait de l'approche partisane de la présidence ${ }^{44}$, la perte de majorité n'a pas atténué la lecture partisane de son statut par le chef de l'État avec un emploi des prérogatives présidentielles à des fins partisanes. Özal a ainsi pu œuvrer tel un opposant politique en usant des prérogatives présidentielles «à plein » comme une faculté d'empêcher ${ }^{45}$.
Cependant, une telle lecture n'est pas systématique puisque l'existence d'une majorité de coalition tempère immanquablement la lecture présidentialisée des institutions. Turgut Özal et Süleyman Demirel ont été successivement deux leaders historiques de la droite qui ont été élus à la présidence. Mais le fait que le premier ait été élu par une majorité confortable composée uniquement de son parti politique alors que le second l'ait été par une majorité de coalition, composée de son parti, mais aussi et surtout du SDP, constituant son principal opposant politique dans sa carrière de Premier ministre durant la II ${ }^{\mathrm{e}}$ République, est révélateur de deux lectures différentes de l'institution présidentielle. Alors que Turgut Özal joue le rôle d'un gouvernant ${ }^{46}$, Süleyman Demirel semble avoir mieux assimilé son rôle d'arbitre malgré son ancrage partisan. Mais cela est surtout dû à une conception très différente de la présidence de la République entre ces deux protagonistes malgré leur volonté commune de réformer l'élection présidentielle en mettant en place une élection au suffrage universel direct. Özal avait un intérêt certain pour le régime présidentiel américain, mais ses propos tendaient vers le rejet d'une séparation stricte des pouvoirs avec la volonté d'éviter les gouvernements de coalition afin d'assurer l'emprise d'un parti unique sur les institutions pour une meilleure gouvernabilité ${ }^{47}$. Demirel souhaitait pour sa part, par la mise en œuvre de l'élection directe, un président qui serait plus impartial ${ }^{48}$, et dont la position se situerait, selon ses propos «entre un président interventionniste et un président dont le rôle se limiterait à la ratification ${ }^{49}$. En effet, Süleyman Demirel voit la présidence comme un frein et contrepoids, une autorité de contrôle, au sein des institutions ${ }^{50}$. Il rejette ainsi une conception purement honorifique, ou "notariale» de la présidence selon ses propres termes ${ }^{51}$, mais admet que celle-ci doit rester

38. Loi no 2533 du 16 octobre 1981 (Resmî Gazete, 16 octobre 1981, 17486 Mükerrer). L'ANAP a été l'un des premiers partis autorisés à exercer une activité politique, alors que les anciens partis, dont notamment le parti de droite de Süleyman Demirel, ont dû attendre le référendum de 1987 pour reprendre leurs activités politiques.

39. Le parti obtient $45 \%$ des voix exprimées dans le pays, ce qui donne lieu à l'élection de 211 députés sur un total de 399 ( $1983-2007$ Y1lları Arasında Yapılan Milletvekili Genel Seçimleri», en ligne: http://www.ysk.gov.tr/tr/1983-2007-yillari-arasi-milletvekili-genel-secimleri/3008).

40. $36 \%$ des voix pour 292 députés ANAP sur un total de 450 députés ( 1983-2007 Yılları Arasında Yapılan Milletvekili Genel Seçimleri»).

41. Il faut noter que l'élection des leaders politiques à la présidence de la République et leur exercice du pouvoir peuvent être parfois ressentis comme un retour à la démocratie, notamment à la suite de la présidence de Kenan Evren. L'élection de Celal Bayar en 1950 par l’intervention du multipartisme à la suite du régime à parti unique et celle de Turgut Özal en 1989, à la suite du mandat de Kenan Evren, peuvent être considérées comme une avancée du pouvoir civil sur l'institution de contrôle qu'est le pouvoir militaire.

42. Voir R. Akın, Gazi'den günümüze..., p. 148.

43. Il faut tempérer ces propos notamment à partir de l'élection de Mesut Yılmaz à la tête de l'ANAP en juin 1991 et dont le gouvernement est resté en place jusqu'à la prise de fonction du gouvernement issu des élections législatives anticipées d'octobre 1991. En effet, Mesut Yılmaz a tenté de limiter l'intervention de la présidence de la République dans la conduite des affaires quotidiennes du gouvernement en imposant son leadership à la tête du parti (voir M. Heper, «Turgut Özal's Presidency: Crisis and the Glimmerings of Consensus », in Politics in the Third Turkish Republic, M. Heper, A. Evin (dir.), Boulder, Westview Press, 1994, p. 195-196).

44. M. Heper, «Turgut Özal's Presidency...», p. 194.

45. Voir R. Akın, Gazi'den günümüze..., p. 148-149.

46. Lors de son arrivée au pouvoir, il nomme YıldırıM. Akbulut comme Premier ministre sans concertation et l'impose à la tête du parti en rétorquant à ceux qui y voit une violation de la neutralité présidentielle qu'il était le fondateur du parti (voir Z. Çağlıyan İçener, «Türkiye'de baskanlık sistemi tartısmalarının yakın tarihi...", p. 320).

47. Voir Z. Çağlıyan İçener, «Türkiye'de baskanlık sistemi tartısmalarının yakın tarihi... », p. 316

48. Ibid., p. 322.

49. Ibid., p. 318

50. Ibid., p. 326

51. Ibid., p. 325. 
au-dessus des contingences partisanes ${ }^{52}$, pour incarner véritablement l'État ${ }^{53}$.

Dans la continuité de la présidence de Süleyman Demirel, celle de l'ancien président de la Cour constitutionnelle A. Necdet Sezer ${ }^{54}$ pose question. Ne provenant pas d'un parti politique, il a pu véritablement incarner cette neutralité présidentielle tant recherchée par ailleurs. Toutefois, sa pratique de la présidence a eu tendance à empêcher la mise en œuvre de la politique gouvernementale. S'est par exemple posée aux juristes turcs, comme ce fut le cas quelques années auparavant en France ${ }^{55}$, la question de savoir si le président pouvait refuser de signer des décrets$\operatorname{lois}^{56}$. En effet, que ce soit avec le gouvernement de coalition mené par Bülent Ecevit ou avec le gouvernement de l'AKP par la suite, A. Necdet Sezer a fait usage de son pouvoir de signature comme d'une modalité de contrôle des actes gouvernementaux. La doctrine est partagée sur le contrôle de juridicité des actes soumis à la signature du président, notamment des décrets-lois pour un renvoi en seconde lecture ${ }^{57}$. Mais, au regard de la fonction de contrôle dévolue au président, le problème est essentiellement de savoir s'il s'agit d'un contrôle juridique ou politique du président. La protection d'un principe comme par exemple celui de la laïcité, dont l'application elle-même est l'objet de clivages entre le président et le parti majoritaire, peut-elle être conçue tantôt comme la protection d'un principe constitutionnel par un organe placé au-dessus des contingences politiques incarnant le pouvoir d'État et tantôt comme un simple objet de régulation politique auquel cas un président neutre ne devrait pas avoir d'opinion à émettre? Le président cherchant à protéger un tel principe agit-il comme une autorité supra-partisane, un gardien de la Constitution, ou comme un simple opposant politique utilisant tous les instruments à sa disposition pour empêcher la majorité titulaire de la fonction gouvernementale de mettre en œuvre sa politique? Ainsi, le problème ne se pose pas simplement sur le contenu des prérogatives présidentielles, mais sur la personne qui les exerce et son mobile. Lorsque ces prérogatives sont exercées dans un mobile partisan, elles relèvent de la fonction gouvernementale de l'État et viennent concurrencer le pouvoir démocratique détenu par la majorité ou la coalition majoritaire. En ce qui concerne la présidence d'A. Necdet Sezer, le fait qu'il ait eu une pratique similaire de ses prérogatives présidentielles alors qu'il a été confronté à une variation de la majorité laisse à penser qu'il a été en adéquation avec la fonction présidentielle telle que déterminée par le constituant. L'enseignement que l'on peut ainsi tirer de la présidence d'A. Necdet Sezer, notamment lorsqu'elle est confrontée à celle d'Abdullah Gül ${ }^{58}$, est que la neutralité présidentielle n'est pas nécessairement en lien avec le caractère passif qui lui est parfois attribué dans certains régimes parlementaires dans lesquels cette fonction peut être purement honorifique ${ }^{59}$. Une présidence partisane, sans l'exercice d'un leadership sur la majorité, crée autant un déséquilibre dans le régime qu'une présidence partisane sous le leadership présidentiel. Le déséquilibre se crée alors au profit de la majorité et de son leader qui est le Premier ministre, le rôle de gardien du chef de l'État n'étant pas pleinement rempli du fait de son absence de neutralité et du caractère passif que prend sa fonction ${ }^{60}$ (voir infra).

Ainsi, le début de la III ${ }^{e}$ République turque a connu des présidences partisanes en pratique malgré le statut neutre imposé par la Constitution. Cela a remis en question l'équilibre des pouvoirs tel que conçu par le constituant de 1982. Du fait de ce renforcement du statut constitutionnel du chef de l'État et de l'immixtion des partis politiques dans le processus de désignation, des épisodes de présidentialisation du régime politique turc ont pu être constatés avant même la mise en place de la réforme de l'élection directe, qui permettra de systématiser une telle lecture.

\section{B. L'intervention de l'élection directe: la systématisation du chef de l'État partisan}

La révision de l'élection présidentielle de 2007 n'est donc pas à l'origine de la lecture partisane du statut du chef de l'État. Toutefois, celle-ci est au fondement de la systématisation de cette lecture par la construction

52. Ibid.

53. «Je suis dans le camp de l'État, dans le camp de la nation, certes naturellement dans le camp du droit et donc je ne suis pas impartial, je le suis politiquement» («Devletin tarafiyım, milletin tarafıyım gayet tabii hukukun tarafiyım ve bu haliyle tarafsiz degilim tarafim; ama siyaseten tarafsızım») (Z. Çağlıyan İçener, «Türkiye'de baskanlık sistemi tartısmalarının yakın tarihi... », p. 325).

54. Présidence du 16 mai 2000 au 28 août 2007.

55. Il est question ici de l'épisode de cohabitation durant lequel le président François Mitterrand a refusé de signer les ordonnances du gouvernement de Jacques Chirac

56. Voir K. Gözler, Cumhurbaşkanı - Hükûmet Çatışması (Cumhurbaşkanı Kararnameleri Imzalamayı Reddedebilir mi ?), ${ }^{\mathrm{re}}$ éd., Bursa, Ekin Kitabevi Yayınlar1, 200o, en ligne: http://www.anayasa.gen.tr/catisma.htm.

57. Selon Bülent Tanör et Necmi Yüzbaşıŏlu, le président peut renvoyer le décret-loi en seconde lecture au Conseil des ministres, mais si celui-ci est adopté dans les mêmes termes par le Conseil, le président n’a plus le choix et doit signer (B. Tanör, N. Yüzbaşığlu, 1982 Anayasasina...,p. 392-393); alors que, pour Kemal Gözler, le président ne peut pas refuser de signer les décrets-lois (K. Gözler, Cumhurbaşkanı - Hükûmet..., p. 31 sq.).

58. Mandat présidentiel du 28 août 2007 au 28 août 2014.

59. L'exercice de la prérogative de renvoi en seconde lecture est particulièrement révélateur d'une telle conception de la présidence. Ainsi, alors qu'A. Necdet Sezer a fait usage de cette prérogative sur plus de soixante-dix textes (en ligne: https://www.tbmm.gov.tr/develop/owa/td_ v2.tutanak_sonuc?v_meclis $=\& \mathrm{v} \_$donem $=\& \mathrm{v} \_$yasama_yili $=\& \mathrm{v} \_$cilt $=\& \mathrm{v} \_$birlesim $=\& \mathrm{v} \_s a y f a=\& \mathrm{v} \_$anabaslik $=$CUMHURBA\%DEKANINCA $\% 20$ GER\%DD\%20G\%D6NDER\%DDLEN\%2oKANUNLAR\&v_altbaslik=\&v_mv=\&v_sb=\&v_ozet=\&v_bastarih=\&v_bittarih=\&v_kayit_ sayisi=200\&v_gelecek_sayfa=101\&v_kullanici_id=10315778), Abdullah Gül n'en aurait fait usage que quatre fois (en ligne : http://www.cumhuriyet. com.tr/haber/turkiye/103255/iste_Gul_un_kosk_karnesi_Geleni_onaylamis.html).

60. Ce qui n'a pas empêché quelques accrochages entre le président Abdullah Gül et le Premier ministre Recep Tayyip Erdoğan, mais davantage sur des questions de forme que de fond (voir S. Yazıcı, «1982 anayasası ve cumhurbaşkanlığı», p. 234). 
d'une légitimité présidentielle contre les autorités de tutelle ayant jusqu'alors une emprise considérable sur le fonctionnement du régime (1). Cet état de fait a permis d'asseoir définitivement le parti majoritaire, sous le leadership présidentiel, au cœur du pouvoir étatique (2).

\section{La cause de la réforme: une réforme par le parti majoritaire contre les autorités de tutelle}

L'élection présidentielle au suffrage universel direct a été longtemps débattue en Turquie. Souhaitée notamment par les figures politiques de la droite telle que Süleyman Demirel et Turgut Özal ${ }^{61}$, cette idée avait été repoussée par les constituants de 1982. En effet, le maintien d'une conception impartiale de la présidence, malgré le renforcement de sa position dans la Constitution de 1982, ne permettait pas la mise en place d'une telle réforme jusqu'en 2007. Mais c'est par le biais de l'affrontement entre les autorités de tutelle incarnant le pouvoir d'État et les institutions représentatives incarnées notamment par le parti majoritaire qu'est l'AKP que la réforme de l'élection directe du président a été mise en œuvre.

Le système politique turc et ses différentes transformations se sont effectivement forgés surtout autour des différentes crises et tensions entre ces deux pouvoirs ${ }^{62}$. L'armée est, dans les faits, la plus active de ces autorités de tutelle, par la constance de son interventionnisme ${ }^{63}$, notamment à la suite des périodes de règne d'un parti majoritaire par les coups d'État successifs ${ }^{64}$. En effet, pensant incarner le pouvoir d'État à travers son idéologie kémaliste, l'armée garde une méfiance accrue envers les partis politiques, surtout de la droite, représentés au sein du gouvernement et du Parlement. Parvenant généralement à rassembler un large électorat dans les périodes de multipartisme et à conserver durant de longues périodes la majorité, ces partis se sont forgés une légitimité politique face à ces périodes de dictatures militaires, particulièrement celle mise en place entre 1980 et 1983 sous l'égide du général Evren, inhibant par la même occasion la force politique des partis de gauche ${ }^{65}$. Une autre de ces autorités de tutelle est incarnée par la Cour constitutionnelle, et ses tensions avec les institutions représentatives se sont illustrées notamment par la dissolution de plusieurs partis politiques.
Mais il ne faut pas oublier que la présidence de la République a également été conçue comme un organe incarnant l'une de ses autorités ${ }^{66}$. Envisagée dans cette perspective, la nécessaire neutralité présidentielle vis-à-vis des partis politiques prend tout son sens, son rôle étant précisément de surveiller le pouvoir démocratique. C'est ce qui explique également le renforcement de son statut et de ses prérogatives lors de l'élaboration de la Constitution de 1982. Cependant, cette position de neutralité a été remise en cause d'abord par les élections de chef de parti politique, et notamment celle de Turgut Özal contestée jusqu'à ses rangs ${ }^{67}$, puis par les effets de la révision de l'élection présidentielle intervenue à la suite de la crise de l'élection présidentielle de 2007 (voir infra).

C'est une fois de plus par l'intervention, cette fois infructueuse, des autorités de tutelle que sont l'armée et la Cour constitutionnelle lors des élections présidentielles de 2007, que l'avenir du régime politique turc s'est scellé. Cette intervention a créé un contexte de crise institutionnelle dont la majorité AKP s'est servie dans le but de légitimer et d'accomplir la révision de l'élection présidentielle au suffrage universel direct tant désirée par les leaders de la droite ${ }^{68}$. Ainsi, lors du premier tour de l'élection présidentielle du 27 avril 2007, la majorité AKP présente son candidat Abdullah Gül, sans avoir préalablement recherché la concertation avec les autres partis comme l'exigeait alors l'esprit de la Constitution de 1982. Alors que les députés des autres formations politiques décident de boycotter ce premier tour, le candidat Abdullah Gül ne parvient pas à être élu en obtenant 357 voix $^{69}$. Ce même jour, les Forces armées turques (TSK) publient un mémorandum sur leur page Internet en s'imposant comme les défenseurs de la laïcité ( 27 Nisan E-Muhtırasi) ${ }^{70}$, et donc en se positionnant implicitement contre l'élection d'Abdullah Gül ${ }^{71}$. À la suite de ce premier tour, les parlementaires de l'opposition saisissent la Cour constitutionnelle, ce qui aboutit à l'annulation du premier tour de l'élection présidentielle au motif que le quorum n'a pas été atteint ${ }^{72}$. Cette position, juridiquement contestable ${ }^{73}$ mais politiquement marquée de la Cour, finit par remettre en question sa légitimité. À la suite de cet épisode, l'AKP procède à des élections législatives anticipées desquelles résulte un renouvellement de la confiance des électeurs avec la

61. Voir E. Onar, «Türkiye’nin başkanlık veya yarı-başkanlık sistemine geçmesi düşünmeli midir?», in Başkanlık Sistemi, T. Ergül (dir.), Ankara, Türkiye Barolar Birliği Yayını, 2005, p. 84-85.

62. Voir A. Kazancigil, "Le système politique turc: mutations démocratiques ou régression islamo-kémaliste?», in Vingt ans de changements en Turquie (1992-2012), J. Marcou, F. Türkmen (dir.), Istanbul - Paris, Université de Galatasaray - L'Harmattan, 2013, p. 14-15.

63. L'armée est déjà intervenue à plusieurs reprises dans le fonctionnement du régime politique successivement en 1960, 1971, 1980, 1994 et 1997 (voir H. Bozarslan, Histoire de la Turquie. De l'empire à nos jours, Paris, Tallandier, 2015, p. 426).

64. Voir M. Sevinç, Türkiye’nin Anayasa imtihanı. Cumhurbaşkanlığı-Başkanlık Tartışması, Istanbul, Iletişim Yayınları, 2017, p. 105.

65. Voir A. Kazancıgil, «Le système politique turc...», p. 15.

66. Voir S. Yazıc1, «1982 anayasası ve cumhurbașkanlığı», p. 231.

67. Voir H. Özdemir, Atatürk'ten günümüze cumhurbaşkanı seçimleri, Istanbul, Remzi Kitabevi, 2007, p. 349.

68. Voir H. Bozarslan, Histoire de la Turquie..., p. 434.

69. La majorité des deux tiers exigée lors du premier tour par l'article 102 de la Constitution n'étant atteinte qu'à 367 voix.

70. Disponible en ligne: http://www.hurriyet.com.tr/gundem/genelkurmaydan-cok-sert-aciklama-6420961.

71. Voir S. Yazıcı, «1982 anayasası ve cumhurbaşkanlığı», p. 243.

72. Anayasa Mahkemesi (Cour constitutionnelle), E. 2007/45, K. 2007/54, K.T. 01/05/2007 (Resmî Gazete, 27 juin 2007, 26565 ).

73. Ni la Constitution, ni le règlement des assemblées n'exigeaient un quorum des deux tiers pour l'élection présidentielle. Par ailleurs, la Cour n'était pas compétente en l'espèce, mais elle est intervenue au titre de sa compétence en matière de contrôle du règlement des assemblées. 
confirmation de sa majorité. Entre-temps, la GANT a déjà adopté la loi de révision constitutionnelle $n^{\circ} 5678$ prévoyant notamment la réforme de l'élection présidentielle au suffrage universel direct, la modification de la durée mandat présidentiel passant du septennat au quinquennat et abaissant la durée de la législature initialement de cinq à quatre ans ainsi que la possibilité de renouveler une fois le mandat présidentiel ${ }^{74}$.

Cette réforme est officiellement présentée comme un instrument de lutte contre les autorités de tutelle et en faveur du règne du pouvoir démocratique. Elle permet officieusement de faire élire comme président le candidat d'un parti sans que sa légitimité ne puisse être contestée par d'autres institutions telles que les autorités de tutelle. La loi de révision constitutionnelle fait alors valoir, non pas un changement institutionnel majeur car le président reste dans la lettre de la Constitution une autorité impartiale vis-à-vis des partis politiques, mais une revalorisation démocratique des institutions ${ }^{75}$ afin d'empêcher tout accaparement par les autorités de tutelle.

Il n'est cependant pas envisageable dans la culture politique turque de mettre en place une élection présidentielle au suffrage universel direct sans que les factions partisanes ne soient sollicitées, voire exacerbées. Les élections présidentielles de 2014 en sont la preuve empirique.

\section{L'effet de la réforme: une réforme pour le parti majoritaire et son leader}

L'objectif inavoué de la réforme est donc aussi de faire du président de la République, non plus une autorité de tutelle, mais bien une autorité gouvernante prenant son ancrage dans une organisation partisane. L'un des aspects de la révision de 2007 consiste à changer les conditions de parrainage en simplifiant les règles de présentation des candidatures non parlementaires à la présidence ${ }^{76}$. Là encore, la possibilité de présenter des candidatures n'est dévolue qu'aux parlementaires, appartenant pour la plupart à des formations politiques ou à une coalition de plusieurs partis politiques non représentés à la GANT. La possibilité de renouveler le mandat par la mise en place d'un quinquennat renouvelable ${ }^{77}$, en lieu et place du septennat non renouvelable prévu initialement par la Constitution de 1982, constitue encore aujourd'hui une atteinte à la neutralité présidentielle et un pas de plus vers la présidence partisane. Mais le fait de réduire à quatre ans la durée de la législature ${ }^{78}$ afin que celle-ci ne soit pas concomitante au nouveau quinquennat présidentiel est un signal pour rassurer les sceptiques sur la question de la neutralité présidentielle ${ }^{79}$. Ainsi, la mise en place de l'élection directe n'a pas donné lieu à la suppression de la mention de la rupture des liens entre le président et son parti d'origine dès l'élection. Le président reste donc soumis en principe à l'obligation d'impartialité imposée par la Constitution de 1982.

Mais le régime politique prend tout de même les aspects d'un régime semi-présidentiel dès l'élection présidentielle qui, en 2014, n'est plus seulement une procédure de désignation du chef de l'État mais aussi une véritable consultation populaire sur la question de la nature du régime politique turc après une telle réforme du mode de désignation du chef de l'État, tant celle-ci est au centre des débats. C'est ce qui conduit plusieurs partis, dont le CHP et le MHP, à faire alliance en présentant une candidature commune en la personne de Ekmeleddin İhsanoğlu ${ }^{80}$. L'objectif est alors de maintenir la lecture parlementaire des institutions en conservant la neutralité présidentielle par le biais d'un candidat qui n'est aucunement un leader partisan.

Comme attendu, l'élection directe ne change pas les rapports entre Recep Tayyip Erdoğan et son parti d'origine. En effet, Abdullah Gül, onzième et dernier président à avoir été élu par la GANT à ce jour, était l'un de membres fondateurs et le numéro deux de l'AKP lors de son accession à la présidence. Mais contrairement à la présidence de Recep Tayyip Erdoğan à partir de 2014, la majorité ne lui était pas personnelle car il n'en était pas le leader, du moins pas le seul, le Premier ministre Recep Tayyip Erdoğan conservant le leadership malgré l'existence de certains clivages. Ainsi, le régime politique turc entre 2007 et 2014 prend davantage les aspects d'un régime primo-ministériel, mais dont la présidence révèle un exercice partisan du pouvoir par la passivité de son contrôle politique, menaçant ainsi l'équilibre constitutionnel dont la condition est la neutralité

74. Resmî Gazete, 16 juin 2007, 26554.

75. Türkiye Büyük Millet Meclisi (Grande Assemblée nationale de Turquie), D. 22, Y.Y. 5, S.S. 1409, p. 7 (en ligne: https://www.tbmm.gov.tr/ tutanaklar/TUTANAK/TBMM/d22/c156/tbmm22156102ss1409.pdf).

76. Article 4 de la loi de révision constitutionnelle $n^{\circ} 5678$ modifiant l’article 101 de la Constitution qui prévoyait dans la rédaction de son alinéa 3 que «la candidature à la présidence de la République nécessite d'être membre de la GANT, ou nécessite, pour les candidatures extérieures à l'Assemblée, la proposition écrite de vingt parlementaires. Par ailleurs, les partis politiques ayant obtenu au total plus de dix pour cent des voix peuvent présenter une candidature commune». Pour rappel, le candidat non parlementaire devait obtenir la proposition écrite d'au moins un cinquième des parlementaires avant la révision de 2007 (voir supra).

77. Article 4 de la loi de révision constitutionnelle $n^{\circ} 5678$ modifiant l'article 101 de la Constitution de 1982.

78. Article 1 de la loi de révision constitutionnelle no 5678 modifiant l'article 77 de la Constitution de 1982.

79. Ce qui a été explicitement souligné dans les motifs du projet de loi de révision soumis à la GANT: «Avec cette modification, le fait de prévoir la réduction à quatre ans des élections de la GANT offre la possibilité au président dont le mandat est de cinq ans de travailler avec des majorités politiques différentes et permet au président élu par le peuple de pouvoir présenter un positionnement impartial, au-dessus des partis politiques " (Türkiye Büyük Millet Meclisi (Grande Assemblée nationale de Turquie), D. 22, Y.Y. 5, S.S. 1409, p. 7).

80. Ekmeleddin Ihsanoğlu est un ancien diplomate turc qui a notamment été le secrétaire général de l'Organisation islamique de coopération. La stratégie politique est de faire prévaloir son expérience à l'international, tout en essayant de capter les électeurs de l'AKP par le recours à un candidat ouvertement musulman (voir G. Perrier, "L'opposition a du mal à faire émerger un candidat crédible à la présidentielle turque», Le Monde, 6 août 2014, p. 3) 
présidentielle. Sous la présidence de Recep Tayyip Erdoğan depuis 2014, la force politique change de camp au sein de l'exécutif. Le Premier ministre ${ }^{81}$ 'a plus de leadership sur la majorité et n'est dans les faits responsable que devant le président. Dès son accession à la présidence, Recep Tayyip Erdoğan, à l'instar de Turgut Özal, désigne seul son Premier ministre sans la concertation de son parti, à la suite de quoi Ahmet Davutoğlu est élu à la tête de l'AKP ${ }^{82}$. Le renvoi de ce dernier en 2016 est le signal non seulement du dualisme instauré dans l'exécutif par l'élection directe, mais aussi d'une proximité entre le président et la majorité, la «démission» du Premier ministre impactant au premier chef l'organigramme du parti ${ }^{83}$. Il y a, à partir de 2014, une pratique anticonstitutionnelle qui s'installe puisque de l'aveu de Recep Tayyip Erdoğan le régime politique a changé de facto $^{84}$. La présidence constante du Conseil des ministres par le président de la République en est une illustration ${ }^{85}$. Le régime politique prend la forme d'un régime semi-présidentiel, mais sans le classique droit de dissolution. Toutefois, le président dispose d'un contrôle total de sa majorité. En suspens lors des législatives de juin 2015, la majorité AKP est finalement confirmée en octobre 2015 à la suite de l'incapacité des partis politiques de former une coalition face au parti présidentiel, tant la polarisation a rendu impossibles certaines alliances. En réalité, la conjugaison d'un président partisan avec une majorité présidentielle a eu pour effet de créer une véritable confusion des pouvoirs. En faisant du gardien de la Constitution, et des prérogatives détenues en conséquence, le chef de la majorité, il en résulte de véritables armes politiques pour le parti majoritaire qui parvient simultanément à réduire à néant les contre-pouvoirs présidentiels pour s'ajouter au pouvoir majoritaire. Cela a été particulièrement exacerbé sous l'état d'urgence mis en place à la suite de la tentative de coup d'État du 15 juillet 2016, avec une pratique abusive des décrets-lois, dont la constitutionnalité et la conventionnalité posent question ${ }^{86}$.
Toutefois, l'objectif pour le président de la République reste la transformation du régime parlementaire en régime présidentiel, notamment pour constitutionnaliser une pratique désormais bien installée: la présidence partisane.

\section{La constitutionnalisation du chef de l'État partisan par la révision de 2017}

Pour justifier la révision, l'exposé des motifs signé par le Premier ministre et les députés AKP pour ce qui concerne spécifiquement la présidence de la République présente la réforme de 2007 comme celle qui a «déjoué le projet tutélaire». Deux profils présidentiels sont mis en opposition: le président «bureaucrate», élu par le Parlement et ayant des prérogatives symboliques, et le président "politique», désigné par le peuple et doté de prérogatives élargies. L'objectif de la réforme de 2017 serait ainsi d'assurer la stabilité politique en rationalisant un régime qui souffrirait d'une forme d'instabilité gouvernementale ${ }^{87}$.

La révision de $2017^{88}$ va tout d'abord intégrer la pratique politique développée depuis la première élection présidentielle au suffrage universel direct en 2014. Mais elle va plus loin en empêchant tout retour à une lecture neutre du statut présidentiel. Car effectivement, et malgré le caractère non obligatoire de l'élection d'un leader partisan à la tête de l'État, il ne fait pas de doute au regard de la culture politique des électeurs turcs, ainsi que des faibles ressources tant politiques qu'économiques des candidats non partisans, que ces derniers n'auraient que très peu de chance d'être élus. Ainsi, la constitutionnalisation du chef de l'État partisan passe d'abord par la pérennisation des acquis de la présidentialisation du régime $(\mathrm{A})$ afin d'empêcher tout retour à la lecture parlementaire de celui-ci et notamment tout retour à la neutralité du chef de l'État (B).

81. Ahmet Davutoğlu (du 29 août 2014 au 24 mai 2016) et Binali Yıldırım (depuis le 24 mai 2016).

82. Le président de parti devant être le chef de gouvernement selon les statuts du parti, officiellement Ahmet Davutoğlu a d'abord été élu à la tête de l'AKP par un congrès extraordinaire du 27 août 2014, puis il a été nommé Premier ministre le 28 (A. Salles, «Le président Erdoğan nomme son "frère Davutoglu” premier ministre", Le Monde, 23 août 2014, p. 4), Recep Tayyip Erdoğan n'ayant pas quitté la tête du gouvernement, ni durant la période de campagne à l'élection présidentielle, ni après son élection le 10 août 2014.

83. En effet, la «démission" a eu lieu par le biais de la non-présentation de candidature de ce dernier à sa propre succession lors du congrès du parti, les statuts prévoyant que le président du parti devait être le chef du gouvernement.

84. C'est ainsi que lors d'une interview sur la chaîne nationale $T R T 1$, le 20 mars 2016, le président Erdoğan a déclaré: «le 10 août [date de la première élection présidentielle], ma nation a fait le choix de ma personne comme président de la République, c'est un régime semi-présidentiel de fait ».

85. En effet, l'article 104 offrait au président de la République la possibilité de présider le Conseil des ministres ou de convoquer le Conseil des ministres sous sa présidence «lorsqu'il le juge nécessaire».

86. İ. Ö. Kaboğlu, «Suppression du régime parlementaire sous l'état d'urgence: remarques sur la modification constitutionnelle "approuvée” par le référendum du 16 avril 2017", Lettre "Actualités Droits-Libertés» de La revue des droits de l'homme, 3 juillet 2017, en ligne: http://journals. openedition.org/revdh/3168.

87. En effet, l'exposé des motifs fait état de plusieurs contradictions, notamment de la question de l'instabilité gouvernementale déclarée à l'aide d'une donnée statistique précisant qu'il y a eu vingt-et-un gouvernements en trente-trois ans. Cela semble paradoxal, comme le souligne Murat Sevinç, pour un parti comme l'AKP qui occupe le pouvoir majoritaire depuis 2002 et détient la présidence depuis 2007, d'évoquer une quelconque instabilité (M. Sevinç Türkiye'nin Anayasa imtihanı..., p. 105).

88. Ne seront pas développés ici les problèmes touchant à la légitimité du référendum et qui ont été dénoncés par ailleurs tels que l'introduction d'une révision constitutionnelle pendant l'état d'urgence ne permettant pas d'instaurer un débat contradictoire (İ. Ö. Kaboğlu, «Suppression du régime parlementaire sous l'état d'urgence... », p. 8), l'absence d'égalité de temps de parole dans les débats et la faiblesse de la participation aux débats des spécialistes opposés à la réforme (K. Gözler, Elveda Anayasa. 16 Nisan 2017'de oylayacağımız anayasa değişikliği hakkında eleştiriler, $2^{\mathrm{e}}$ éd. Bursa, Ekin, 2017, p. 29-42), les irrégularités de procédure durant le vote des députés (M. Sevinç, Türkiye’nin Anayasa imtihanı..., p. 106-108) et durant le référendum (İ. Ö. Kaboğlu, «Suppression du régime parlementaire sous l'état d'urgence...», p. 14). 


\section{A. La pérennisation des acquis de la présidentialisation du régime}

L'organisation des pouvoirs telle qu'elle découle de la révision constitutionnelle de 2017 est inédite par rapport aux modèles de référence en matière de classification des régimes politiques. Depuis la tentative d'élaboration de la Constitution dite civile ${ }^{89}$ débutée en juin 2011 avec la mise en place d'une Commission de conciliation constitutionnelle à la GANT, l'AKP a toujours revendiqué son désir d'instituer un régime présidentiel. Mais l'évolution du projet, et certaines différences fondamentales avec le régime de séparation stricte des pouvoirs, ont eu pour conséquence la requalification du projet en «régime présidentiel à la turque» pour finalement aboutir en 2016 à la présentation d'un nouveau type de «régime de gouvernement de la présidence de la République» (Cumhurbaşkanliğı hükümet sistemi). En effet, il apparaît difficile de classer le projet adopté dans l'une ou l'autre catégorie de la classification classique des régimes politiques constituée par le régime présidentiel et le régime parlementaire, d'où une forme d'originalité dans l'appellation de ce régime. Le principe de séparation des pouvoirs, essentiel à cette classification, fait clairement défaut dans l'ingénierie de cette révision (1), et la duplicité de la fonction présidentielle qui, tout en conservant les prérogatives d'un chef de l'État parlementaire neutre, acquiert celles d'un Premier ministre leader de la majorité au pouvoir, a un effet néfaste sur l'équilibre des pouvoirs en inhibant tout élément pouvant faire office de contre-pouvoir. Cette duplicité existait par la mainmise présidentielle sur la fonction du Premier ministre qui a été constatée lors de la première partie de la présidence de Turgut Özal, et aussi depuis 2014. Elle apparaît désormais formalisée (2). Ces deux éléments concourent ainsi à une confusion des pouvoirs, préalablement installée par la pratique politique, et consolidée par la stature partisane du chef de l'État.

\section{Un confusionnisme des pouvoirs législatif et exécutif entretenu par la présidence partisane}

L'organisation des pouvoirs législatif et exécutif relève de deux modèles classiques de référence que sont le régime parlementaire et le régime présidentiel. Le critère permettant de distinguer efficacement le régime présidentiel du régime parlementaire est constitué par l'absence de moyen d'action entre les pouvoirs permettant à l'un des pouvoirs d'écourter le mandat de l'autre ${ }^{90}$. C'est sur ce critère qu'il est possible d'instaurer une distinction logique entre ces deux modèles ${ }^{91}$. Or, la révision constitutionnelle adoptée par le référendum du 16 avril 2017 prévoit précisément ce type de mécanisme entre le législatif et l'exécutif impliquant le rejet du régime présidentiel. L'article $11 \mathrm{du}$ projet de révision adopté par la GANT modifie l'article 116, alinéa 2 attribuant au président le pouvoir de provoquer de nouvelles élections législatives s'assimilant sur le principe à un droit de dissolution de la GANT. Mais l'usage de cette prérogative implique également la remise en jeu du mandat présidentiel, conformément à la logique instituée par la disposition imposant le principe de la simultanéité des élections législatives et présidentielles ${ }^{92}$. Le premier alinéa de l'article 116 attribue également à la GANT la prérogative de renouveler les élections législatives et présidentielles mais exige une majorité qualifiée de trois cinquièmes des députés. Ainsi, l'on retrouve ici formellement une interdépendance organique des pouvoirs, même s'il faut noter un net déséquilibre au profit du président de la République ${ }^{93}$. Le régime présenté sous les traits du régime présidentiel se rapprocherait donc davantage du modèle parlementaire au regard du critère de l'interdépendance organique. Cependant, au vu de l'organisation de celui-ci, il s'agit bien ici d'un régime parlementaire atypique, voire déformé ${ }^{94}$. En effet, la possibilité de renouvellement des élections ne se confond pas avec le critère que certains auteurs estiment essentiel pour définir le régime parlementaire qui est la responsabilité gouvernementale devant le Parlement ${ }^{95}$. Présenté à la Commission de Venise comme un mécanisme de freins et contrepoids ${ }^{96}$, ce processus de « renouvellement bilatéral» permettrait d'avoir recours à l'arbitrage populaire et de parer à tout blocage institutionnel ${ }^{97}$. Le but de celui-ci n'est donc pas de créer un équilibre mais d'assurer que l'exécutif ait la même vision politique que le législatif, ce qui s'inscrit dans la logique du régime parlementaire. Toutefois, dans l'esprit de ce dernier, c'est en principe le Parlement qui doit s'assurer que le gouvernement se

89. En opposition à la Constitution de 1982 adoptée à la suite du coup d'État de 1980 .

90. Richard Moulin se réfère ainsi à Léo Hamon : «Le régime présidentiel est celui où chacun des pouvoirs est sans action sur la naissance et la durée du mandat de l'autre» (R. Moulin, Le présidentialisme et la classification des régimes politiques, Paris, LGDJ, 1978, p. 25).

91. En effet, selon Michel Troper, reprenant en la matière le raisonnement de Charles Eisenmann (C. Eisenmann, «Essai d'une classification théorique des formes de gouvernement ", in Écrits de théorie du droit, de droit constitutionnel et d'idées politiques, C. Leben (dir.), Paris, Presses de l'université de Panthéon-Assas (Les introuvables), 2002, p. 331), la valeur logique d'une classification suppose "que les classes s'opposent trait pour trait», ainsi le critère définissant l'une des classes doit être absent dans l'autre classe (M. Troper, «Les classifications en droit constitutionnel», Revue du droit public, n' 4 , juillet-août 1989, p. 947).

92. Article 4 de la loi de révision constitutionnelle n 6771 modifiant l'article 77 de la Constitution de 1982 (Resmî Gazete, 11 février 2017, 29976).

93. Il est plus aisé de déclencher le renouvellement des élections pour le président (c'est un pouvoir discrétionnaire car il n’y a pas de condition formelle à respecter) que pour la GANT (qui doit réunir une majorité qualifiée)

94. Voir K. Gözler, Elveda Anayasa..., p. 15-16.

95. Voir J.-C. Colliard, Les régimes parlementaires contemporains, Paris, Presses de la Fondation nationale des sciences politiques, 1978, p. 18-19.

96. Commission européenne pour la démocratie par le droit (ci-après «Commission de Venise»), 13 mars 2017, Avis sur les modifications de la Constitution adoptées par la Grande Assemblée nationale le 21 janvier 2017 et soumises au référendum national le 16 avril 2017 , adopté par la Commission de Venise à sa $110^{e}$ session plénière (Venise, 9-11 mars 2017), Avis nº 875/2017, p. 21.

97. Selon l'exposé des motifs de la proposition de révision constitutionnelle (Türkiye Büyük Millet Meclisi (Grande Assemblée nationale de Turquie), D. 26, Y.Y. 2, S.S. 447, p. 14, en ligne: https://www.tbmm.gov.tr/sirasayi/donem26/yilo1/ss447.pdf). 
conforme bien à son orientation politique et mette en jeu sa responsabilité par le biais d'une motion de censure si ce n'était pas, ou plus, le cas ${ }^{98}$. Ce procédé est donc conforme à la logique parlementariste dans son principe d'identité de l'orientation politique de l'exécutif et du législatif, mais la logique y est inversée dans la mise en œuvre des moyens permettant cette identité. En mettant en place une interdépendance organique par la possibilité de provoquer le renouvellement des élections, notamment au profit du président, avec un exécutif monocéphale et sans responsabilité politique véritable, le constituant entend constitutionnaliser la présidentialisation amorcée depuis les élections présidentielles de 2014 en lui donnant les moyens de disposer d'une majorité portant ses couleurs politiques. L'ingénierie constitutionnelle, par l'organisation simultanée des élections législatives et présidentielle, favorise ainsi la discipline de parti. Mais dans un pays dans lequel l'organisation intra-partisane des différents partis est aussi oligarchique et hiérarchisée ${ }^{99}$, cette simultanéité a pour effet d'élire systématiquement une majorité docile au président de la République. Et c'est précisément cette donnée qu'est la discipline de parti qui finit par parachever la confusion des pouvoirs mise en place par cette révision. En effet, sauf circonstances exceptionnelles, l'orientation politique de la personnalité élue à la présidence est similaire à celle de la majorité parlementaire. La simultanéité des élections apparaît comme une garantie pour assurer au président une majorité personnelle alors que son élection directe lui procure la légitimité nécessaire à construire son leadership, si celui-ci lui fait défaut, sur cette majorité $^{100}$. Dans l'hypothèse, très peu probable, où l'élection aboutirait à une opposition politique entre le législatif et l'exécutif, le renouvellement anticipé des élections reste un instrument permettant de contourner un éventuel blocage du législatif pour le président. Tout est mis en œuvre afin d'éviter l'hypothèse d'une cohabitation, conformément à une logique parlementariste.

Au-delà des rapports organiques, la spécialisation fonctionnelle apparaît comme relative dans la réforme constitutionnelle. À cet égard, la fonction législative est aussi symptomatique de cette confusion des pouvoirs. L'absence d'initiative présidentielle est bienvenue par rapport à l'irresponsabilité politique présidentielle. Toutefois, il est fort probable, au regard de la position présidentielle sur sa majorité, que le président n'éprouve aucune difficulté à « inviter» les parlementaires de sa majorité à proposer les réformes désirées ${ }^{101}$. Il ne s'agirait pas ici d'une «démocratie de couloir » à l'américaine, la discipline de parti prenant le pas, dans ce système, sur la négociation et le compromis entre l'exécutif et les représentants composant la Chambre américaine. Par ailleurs, la campagne préalable aux élections simultanées de la présidence et des parlementaires est menée sous l'égide d'un programme unique pour les candidats à la présidence et ceux à la députation. La probabilité que le président soit également le chef de parti de la majorité, et pouvant à ce titre s'exprimer devant les instances du parti, étant forte, les discours devant les instances du parti peuvent être l'occasion pour le chef de l'État d'impulser les réformes législatives souhaitées ${ }^{102}$. En outre, en tant que chef de l'État il dispose du droit de message "à propos de la politique intérieure et extérieure du pays » en vertu de la nouvelle rédaction de l'article 104, alinéa 4, mais aussi, «lorsqu'il le juge utile, il peut faire un discours d'ouverture le premier jour de la session parlementaire » en vertu du troisième alinéa du même article. Tous ces outils ont le potentiel d'une prérogative d'impulsion tant que la GANT a la même orientation partisane que le président. Enfin, le président détient une arme conséquente avec le veto présidentiel que la GANT ne peut surmonter qu'à la majorité absolue du nombre total de députés ${ }^{103}$, ce qui paraît peu vraisemblable au regard de la probable organisation des forces en présence du fait de la simultanéité des élections. Le président reste donc impliqué dans la fonction législative, mais c'est essentiellement par le biais de sa stature partisane.

Concernant la fonction exécutive, avec la suppression du Premier ministre et du gouvernement, celle-ci se trouve entièrement dévolue au président de la République ${ }^{104}$. Pour ce faire, le président a recours aux décrets présidentiels qui sont sources de questionnement quant au caractère flou, et donc potentiellement large, de leur champ d'application $^{105}$. Il apparaît dans la rédaction de l'alinéa 17 de l'article 104 que les décrets présidentiels constituent un pouvoir réglementaire autonome puisque leur champ d'application n'est défini que négativement ${ }^{106}$. Bien que la primauté soit explicitement accordée à la loi, celle-ci

98. Voir D. Baranger, A. Le Divellec, «Régime parlementaire», in Traité international de droit constitutionnel, t. II, Distribution des pouvoirs, D. Chagnollaud, M. Troper (dir.), Paris, Dalloz, 2012, p. 172-173. Conformément à la pratique politique mise en œuvre depuis 2014.

99. Voir M. Yanık, Liberal Perspektif Rapor, $\mathrm{n}^{\circ}$ 5, janvier 2016, Türkiye'de siyasi partiler ve demokrasi, rapport pour l'association Özgürlük araştırmaları derneği, p. 14 .

100. Ce qui ne paraît pas envisageable dans la mesure où, au regard des prérogatives présidentielles et de son rôle dans le régime politique, le candidat à l'élection présidentielle pour chaque parti sera son leader politique. La légitimité tirée de l'élection directe ne fera que consolider le poids du président sur son parti.

101. Conformément à la pratique politique mise en œuvre depuis 2014.

102. C'est le cas actuellement, puisque le président, depuis l'entrée en vigueur de la suppression de la mention à l'article 101 faisant référence à la rupture des liens entre le président et son parti politique, préside notamment les réunions de groupe de son parti à la GANT, dont les discours sont désormais relayés par le site officiel de la présidence de la République (https://www.tccb.gov.tr/receptayyiperdogan/konusmalar).

103. Article 89 de la Constitution de 1982 dans sa version modifiée par l'article $16 \mathrm{C}$ de la loi de révision constitutionnelle $\mathrm{n}^{\circ} 6771$ (Resmî Gazete, 11 février 2017, 29976).

104. Article 104, alinéa $1^{\text {er }}$ de la Constitution de 1982 (Resmî Gazete, 11 février 2017, 29976).

105. Voir İ. Ö. Kaboğlu, «Suppression du régime parlementaire sous l'état d'urgence... », p. 9.

106. Sont ainsi exclus les domaines suivants: les droits fondamentaux, les droits et devoirs de l'individu prévus dans les deux premiers chapitres de la deuxième partie de la Constitution et les droits et devoirs politiques prévus dans le quatrième chapitre (les droits économiques et sociaux 
risque d'être compromise selon İlyas Doğan par le droit de veto que peut exercer la présidence sur les lois contredisant son décret présidentiel ${ }^{107}$. Mais là encore, et sans même que le président n'ait à faire usage de son droit de veto, la composition politique de la GANT peut aboutir à une inhibition du pouvoir législatif et laisser le champ libre aux décrets présidentiels. Par ailleurs, la proclamation de la nullité des décrets présidentiels intervenant dans les domaines cités par la Constitution, et notamment dans le domaine législatif, dépend en grande partie du contrôle exercé par les juges sur ces actes, et donc de leur indépendance vis-à-vis du pouvoir exécutif ${ }^{108}$ (voir infra).

La confusion des pouvoirs législatif et exécutif constitue une donnée assez récurrente dans les régimes parlementaires actuels causée essentiellement par le système de parti. Pour remédier à cet état de fait et répondre à la critique de la transformation des chambres législatives en chambres d'enregistrement, la fonction dévolue aux assemblées représentatives n'est plus seulement présentée comme une fonction normative, mais aussi, et essentiellement, comme une fonction de contrôle. C'est le développement de cette dernière qui marque le mouvement de démocratisation des régimes parlementaires actuels ${ }^{109}$. Toutefois, la révision de 2017 ne laisse que peu de place à l'exercice d'un tel contrôle, le peu de mécanisme existant étant neutralisé par la discipline de parti (voir infra). En effet, puisque le poste de Premier ministre et le gouvernement sont supprimés, il n'y a plus ni vote de confiance ni motion de censure ${ }^{110}$. Les ministres et vice-présidents ne sont responsables que devant le président ${ }^{111}$. De plus, le pouvoir présidentiel en matière budgétaire s'élargit, alors qu'il s'agit ici d'un domaine classiquement dévolu à l'organe législatif en régime parlementaire comme en régime présidentiel, permettant un contrôle efficace du pouvoir législatif sur l'exécutif ${ }^{112}$.

En matière de responsabilité pénale du président ${ }^{113}$, il est possible pour la GANT d'introduire une proposition d'enquête parlementaire mais elle ne peut être initiée qu'à la majorité absolue du nombre total de députés et adoptée à la majorité des trois cinquièmes du nombre total de députés. Si cette majorité est réunie, l'instruction est menée par une commission de quinze parlementaires issus des différents partis politiques représentés à la GANT et proportionnellement à leur nombre après tirage au sort. La commission transmet son rapport qui est examiné en séance plénière. La GANT peut saisir la Haute Cour par une majorité des deux tiers qui statuera sur le sort du président. Bien que la saisine de la Haute Cour exige une moindre majorité depuis la révision de 2017 (une majorité de trois quarts du nombre total des députés était exigée auparavant ${ }^{114}$ ), l'initiative de l'enquête parlementaire s'est davantage complexifiée (l'initiative étant dévolue à un tiers avant la révision de 2017, alors que la proposition d'enquête doit être adoptée à une majorité des trois cinquièmes difficile à atteindre). Malgré le caractère très exceptionnel de la mise en œuvre d'une telle procédure et donc la nécessité de mettre en place des majorités qualifiées, la faiblesse des moyens de contrôle de l'action présidentielle aurait pu être compensée par la possibilité pour la minorité parlementaire de faire entendre sa voix à travers ce type de procédure. L'absence de responsabilité politique de l'exécutif est une contradiction de ce régime mis en place, et l'émergence de celle-ci, fusse à travers l'initiation d'une proposition d'enquête parlementaire, aurait pu constituer un moyen pour l'opposition parlementaire de faire entendre sa voix. En effet, un fonctionnement similaire au déclenchement d'une motion de censure pourrait être instauré par usage, et, malgré ses faibles chances de réussite quand elle est lancée par une minorité, pourrait potentiellement occuper le temps politique et interpeller le pouvoir. Dans un régime dans lequel des procédures de mise en jeu de la responsabilité politique existent, une procédure complexe de mise en jeu la responsabilité pénale s'impose, l'enjeu étant de distinguer les deux formes de responsabilité. Mais dans le cadre d'un régime dans lequel les contrepouvoirs sont inexistants ou neutralisés, et l'absence de responsabilité politique constitue une anomalie, une telle procédure peut permettre de faire entendre une voix discordante et pallier ainsi l'absence de responsabilité politique. Au regard de la stature partisane du président et de la présence de sa majorité, la probabilité qu'une telle

prévus dans le troisième ne sont pas cités, impliquant la possibilité de les réguler pour les décrets présidentiels); les domaines dans lesquels la Constitution consacre la compétence exclusive de la loi; les domaines dans lesquels la loi est expressément intervenue; les domaines dans lesquels on retrouve des dispositions contradictoires entre le décret présidentiel et la loi (ce sont alors les dispositions législatives qui s'appliquent); si la GANT légifère dans le même domaine que le décret présidentiel, celui-ci devient nul.

107. I. Doğan, «Türk tipi başkanlık modeli bağlamında 2017 Anayasa değişikliğinin irdelenmesi », in Dört kıtada başkanlık sistemi, İ. Doğan, S. Ünver (dir.), Ankara, Astana Yayınları, 2017, p. 29.

108. Voir K. Gözler, Elveda Anayasa..., p. 62-63.

109. C'est par exemple la position de Xavier Vandendriessche concernant la France («Le Parlement entre déclin et modernité », Pouvoirs, n 99 , 2001, p. 62-64).

110. Article 6 de la loi de révision constitutionnelle no 6771 modifiant l'article 98

111. Selon la nouvelle rédaction de l'article 106, alinéa 5 modifié par l'article 10 de la loi de révision constitutionnelle $n^{\circ} 6771$. Seule la nouvelle rédaction de l'article 98, en permettant aux parlementaires de poser des questions écrites aux ministres et vice-présidents, amorce l'idée d'un certain contrôle du législatif sur l'exécutif (article 6 de la loi de révision constitutionnelle ${ }^{\circ} 6771$ ).

112. Ainsi, conformément à l'article 161, alinéa 3 , il détient l'initiative budgétaire (article 15 de la loi de révision constitutionnelle nº 6771 ), mais surtout, par l'alinéa 4, en cas de retard dans l'adoption de la loi de finance, il faut adopter une loi de finance provisoire. Mais si cette dernière n'était pas adoptée non plus, c'est le budget de l'année précédente, affecté d'un taux d'actualisation déterminé par la loi, qui entrerait en vigueur.

113. Article 105 modifié par l'article 9 de la loi $n^{\circ} 6771$. Une procédure similaire est prévue pour les vice-présidents et les ministres (article 106, alinéa 5 sq., modifié par l'article 10 de la loi n 6771).

114. Version initiale de l'article 105 applicable jusqu'à l'entrée en vigueur de la révision de 2017 (Resmî Gazete, 9 novembre 1982, 17863, 1. Mükerrer, p. 29). 
procédure soit enclenchée est quasiment nulle. Il est donc nécessaire de développer en contrepartie des mécanismes s'adressant à l'opposition parlementaire.

L'agencement des pouvoirs entre le législatif et l'exécutif souffre donc de nombreuses incohérences et a pour effet d'affaiblir la GANT. Mais c'est la stature partisane du président qui permet de mettre en œuvre une confusion entre ces deux pouvoirs. Celle-ci est entretenue par un autre élément clé de la réforme qui est la duplicité de la fonction présidentielle, provenant de l'absence d'un exécutif bicéphale ${ }^{115}$.

\section{La duplicité de la fonction présidentielle}

La monocéphalisation de l'exécutif constitue un élément clé de la déviance présidentialiste des régimes parlementaires dont l'exécutif est classiquement bicéphale ${ }^{116}$. Celleci s'opère globalement par la prééminence présidentielle sur l'exécutif, et notamment sur le chef de gouvernement, confortée par l'existence d'une majorité personnelle au Parlement. La maîtrise de sa majorité par le président partisan $^{117}$ a eu pour effet de soumettre le poste de Premier ministre à la volonté présidentielle en inhibant, dans les faits, le bicéphalisme de l'exécutif. La révision de 2017 constitutionnalise ainsi le monocéphalisme du régime amorcé dès 2014 et renforcé par le renvoi du Premier ministre Ahmet Davutoğlu sous la forme d'une démission forcée ${ }^{118}$ confirmant que le gouvernement n'était responsable en fait que devant le président ${ }^{119}$. En supprimant le poste de Premier ministre et l'entité collégiale et solidaire qu'est le gouvernement en régime parlementaire par cette dernière révision, le chef de l'État devient le seul titulaire de l'ensemble de la fonction exécutive, fonction elle-même renforcée, entre autres, par la mise en place des décrets présidentiels (voir supra). Mais il conserve dans le même temps les prérogatives qui lui sont dévolues en tant que chef de l'État neutre et qui touchent aux autres fonctions, notamment à la fonction législative et juridictionnelle. Ainsi, la révision constitutionnelle de 2017 , bien que d'une ampleur inédite, juxtapose une présidence partisane à un régime conçu comme parlementaire avec un chef de l'État neutre ${ }^{120}$. La conjugaison de ces deux types de présidence, dont le statut était déjà renforcé par rapport aux canons du parlementarisme classique, a pour effet d'anéantir tout équilibre des pouvoirs.

Cette duplicité de la fonction présidentielle devient ainsi préoccupante au regard de ses prérogatives liées à la fonction juridictionnelle. La consécration du président gouvernant et potentiellement partisan lors de la révision de 2017 aurait dû avoir pour conséquence de revoir le mode de désignation des organes en charge de la fonction juridictionnelle afin de leur conférer la plus grande indépendance par rapport au pouvoir politique. En effet, la Constitution de 1982 a attribué un pouvoir de nomination important au président neutre. L'article 104 prévoit initialement que les prérogatives du président en matière juridictionnelle comprennent la désignation des «membres de la Cour constitutionnelle, le quart des membres du Conseil d'État, le procureur général de la République près de la Cour de cassation, le procureur général de la République adjoint près de la Cour de cassation, les membres de la Cour de cassation militaire, les membres du Haut Tribunal administratif militaire, les membres du Conseil supérieur des juges et des procureurs ». La révision constitutionnelle de 2010 adoptée par le référendum du 12 septembre de la même année ${ }^{121}$ a modifié les règles de nomination pour diminuer ce pouvoir de nomination du président. Alors que le président nommait l'ensemble des onze titulaires et quatre suppléants de la Cour constitutionnelle parmi les candidats proposés par différentes juridictions, il désigne à partir de 2010 quatorze des dix-sept membres, le reste relevant de la GANT ${ }^{122}$. Concernant le Haut Conseil des juges et des procureurs (HSYK), sa composition a également été modifiée en 2010. Initialement, en plus du ministre de la Justice et de son secrétaire d'État, le président désignait trois titulaires et autant de suppléants parmi les candidats présentés par la Cour de cassation et en leur sein, et deux titulaires et autant de suppléants parmi les candidats présentés par le Conseil d'État et en leur sein. En 2010, le Haut Conseil est composé de vingt-deux titulaires et douze suppléants. Parmi les titulaires, outre le ministre de la Justice et son

115. İbrahim Ö. Kaboğlu résume ainsi la situation: «En bref, une personne sera titulaire de trois présidences: le parti, le gouvernement, l’État» ( Suppression du régime parlementaire sous l'état d'urgence...», p. 9).

116. Selon Georges Vedel, «la dualité des fonctions de chef de l'État d'une part et du cabinet de l'autre est un des traits propres du régime parlementaire [...]. En revanche, c'est le cumul des fonctions de chef de l'État et de chef de gouvernement qui caractérise le régime présidentiel» (G. Vedel, Manuel élémentaire de droit constitutionnel, Paris, Recueil Sirey, 1949, p. 426; cité par R. Moulin, Le présidentialisme..., p. 53).

117. L'une des illustrations de l'influence présidentielle sur le parti face au Premier ministre est l'épisode par lequel les proches du président et membres de l'instance dirigeante ont retiré au président du parti et chef de gouvernement qu'était alors Ahmet Davutoğlu la prérogative qu'il détenait en conséquence de nommer les cadres régionaux du parti (M. Jégo, «En Turquie, la disgrâce d'un Premier ministre», Le Monde, 7 mai 2016 , p. 3 ; «Tensions avec Erdoğan: le sort du Premier ministre turc Davutoğlu très incertain », France 24, 4 mai 2016, en ligne: http://www.france24.com/ fr/20160504-tensions-erdogan-davutoglu-akp-turquie-visas-turcs-nomination-ankara-president-allah).

118. M. Jégo, «En Turquie, la disgrâce...», p. 3.

119. Ainsi, selon la première partie de l'article 106, alinéa 5 telle que révisée par l'article 10 de la loi de révision constitutionnelle ${ }^{\circ} 6771$ : «Les viceprésidents et ministres sont responsables devant le président».

120. La Commission de Venise a exprimé ses doutes sur cette conception de la présidence de la manière suivante: «La Commission de Venise doute qu'un président étroitement lié à un parti puisse assumer ce rôle et être perçu comme l'assumant vraiment. On voit mal comment, s'il est affilié à un parti et détient l'intégralité du pouvoir exécutif, il pourra en même temps être un président symbolique et politiquement neutre. La révision ne choisit pas entre ces deux options contradictoires " (Commission de Venise, Avis nº 875/2017, p. 13).

121. Loi de révision constitutionnelle no 5982 du 7 mai 2010 (Resmî Gazete, 13 mai 2010, 27580).

122. Article 146 de la Constitution de 1982 modifié par l'article 16 de la loi $n^{\circ} 5982$. 
secrétaire d'État, le président désigne quatre membres parmi les enseignants du supérieur spécialisés dans les branches du droit et les avocats. Les autres membres sont désignés par les hautes juridictions (cinq), les juridictions ordinaires (dix) et l'Assemblée générale de l'Académie de justice turque (un). Un tel pouvoir de nomination, malgré un affaiblissement, exige une neutralité certaine de la part de la présidence, ce qui était présent dans l'esprit et la lettre de la Constitution de 1982 dans sa version initiale (voir supra). La révision de ces procédures de nomination était nécessaire au regard du principe de séparation des pouvoirs et de l'indépendance de la justice. Si cela n'était pas assuré, un pouvoir de contrôle sur ces nominations aurait dû être attribué aux représentants de la GANT ${ }^{123}$ malgré le facteur de la discipline partisane qui systématiserait un tel vote de la part des députés de la majorité présidentielle. Or, la révision de 2017 ne prévoit pas une modification de grande ampleur concernant la Cour constitutionnelle puisque seuls les sièges attribués aux membres désignés au sein et parmi les propositions des juridictions militaires sont supprimés, ces dernières étant elles-mêmes supprimées ${ }^{124}$. Désormais, la Cour constitutionnelle est donc composée de quinze membres dont douze sont désignés par le président et trois par la GANT. Le Haut Conseil des juges et des procureurs change de dénomination et devient le Conseil des juges et des procureurs, mais c'est surtout la nouvelle composition de l'institution qui éveille les craintes quant à son indépendance ${ }^{125}$. Alors que dans le régime précédent, malgré la nomination de certains membres par le président, la grande majorité des membres étaient nommés par leurs pairs, cette prérogative appartient désormais exclusivement au pouvoir politique $\mathrm{e}^{126}$ : le président désigne six membres (dont les membres de droit que sont le ministre de la Justice et son secrétaire d'État) et il revient à la GANT d'en élire sept ${ }^{127}$. L'exposé des motifs par article présente cette révision comme un «renforcement de la légitimité démocratique ${ }^{128}$. Toutefois, la probabilité de la haute teneur politique de l'institution, mais aussi et surtout, de l'absence de pluralité parmi les membres est un élément confirmant la thèse d'une confusion inédite des pouvoirs, et, notamment, l'influence sans pareille que détient désormais le président sur ce Conseil. En effet, en plus de la disposition relative au statut partisan, la disposition tenant à la recomposition du Conseil des juges et des procureurs fait partie de celles qui sont immédiatement entrées en vigueur après le référendum démontrant l'empressement du pouvoir à ce sujet.

Le retour à la neutralité présidentielle pourrait tempérer ce qui a été vu précédemment et permettre d'amorcer, à nouveau, une forme de séparation des pouvoirs. Car, en effet, la suppression de la mention prescrivant au président de rompre les liens avec son parti ne constitue pas un impératif pour les électeurs les empêchant d'élire un président non partisan. Mais en l'état actuel du régime et de la culture politique de la société, des institutions et des partis politiques, l'élection d'une personnalité non partisane, exerçant la fonction présidentielle de manière impartiale, serait-elle plausible?

\section{B. L'impossible retour à la neutralité présidentielle?}

L'hypothèse de l'élection d'une personnalité non partisane n'est juridiquement pas exclue. Cependant, celle-ci est politiquement peu probable tant la réforme accentue la logique partisane de cette élection (1). En effet, il a été vu précédemment que malgré la consécration de la neutralité présidentielle dans son principe même et par la régulation de son statut en 1982, l'influence partisane sur l'élection présidentielle s'est toujours fait ressentir durant les élections indirectes par la GANT, et celle-ci s'est imposée dès l'élection présidentielle au suffrage universel direct de 2014. La révision de 2017 enterre définitivement toute hypothèse de retour à la neutralité présidentielle. L'une des issues pour assurer des mécanismes de contre-pouvoirs consisterait à développer une démocratie intra-partisane qui ne semble pas acquise dans la culture des partis politiques turcs dont l'organisation est en général oligarchique et fortement hiérarchisée (2).

\section{L'accentuation de la logique partisane de l'élection présidentielle}

La simultanéité des élections, conjuguée à la possibilité pour le président d'être également président de parti, va probablement donner lieu à une candidature à la présidentielle des chefs de parti. L'intention actuelle des partis les plus importants semble pencher vers la candidature des leaders de majorité et non vers des candidats représentatifs d'une neutralité comme peuvent l'être un ancien juge constitutionnel ou un haut fonctionnaire.

L'évolution des dispositions juridiques concernant les conditions de présentation des candidatures illustre ainsi l'acceptation par le droit de la pratique politique tenant à l'influence des partis sur les élections présidentielles depuis la révision 2007, puis un accroissement de celle-ci avec la révision de 2017. Dans sa version antérieure, la Constitution prévoyait un parrainage par vingt parlementaires ou la possibilité pour les partis ou une coalition

123. Comme c'est le cas aux États-Unis où le Sénat américain contrôle les nominations présidentielles par le biais de sa prérogative de confirmation en vertu du deuxième alinéa de l'article II, section II.

124. Article $17 \mathrm{E}$ de la loi de révision constitutionnelle $\mathrm{n}^{\circ} 6771$.

125. Commission de Venise, Avis no 875/2017, p. 22.

126. Voir E. Güner Toprak, Çare başkanlık $m \imath$ ?, $7^{\mathrm{e}}$ éd., Ankara, Palme Yayıncılık, 2017, p. 107-112.

127. Article 14 de la loi de révision constitutionnelle $n^{\circ} 6771$ modifiant l'article 159 de la Constitution de 1982

128. Türkiye Büyük Millet Meclisi (Grande Assemblée nationale de Turquie), D. 26, Y.Y. 2, S.S. 447, p. 14, en ligne: https://www.tbmm.gov.tr/sirasayi/ donem26/yilo1/ss447.pdf. 
de partis de présenter une candidature commune s'ils parvenaient à cumuler plus de $10 \%$ des suffrages de la précédente élection. L'article 101 offre désormais aux partis politiques qui ont obtenu, seuls ou en coalition, plus de $5 \%$ des suffrages aux dernières élections générales la possibilité de présenter un candidat à la présidence de la République. Un parrainage populaire est également mis en place par la révision permettant à cent mille électeurs de présenter leur propre candidat aux élections présidentielles, ce qui constitue indéniablement une avancée démocratique conformément à l'objectif affiché dans l'exposé des motifs visant à «accroître la participation démocratique ${ }^{129}$. Cependant, il s'agit bien d'un recul, voire d'un coup d'arrêt, à la neutralité présidentielle, impactant l'équilibre des pouvoirs. L'objectif affiché dans l'exposé des motifs concernant la présentation des candidats par le parti est bien «d'accroître l'influence des partis politiques ». La simplification des règles de présentation des candidatures dénote une volonté d'exacerber la compétition politique autour d'un organe dont l'essence est la neutralité, et qui, pour assurer un tel objectif, doit être désigné par la voie du consensus.

Le caractère renouvelable du mandat présidentiel ${ }^{130}$ est également un facteur qui accentue la logique partisane de l'élection puisque cela incite le président remettant en jeu son mandat et les candidats de l'opposition à mettre en place une campagne programmatique autour de l'action présidentielle. Dans la continuité de la révision de 2007 , la révision de 2017 maintient la limitation à deux mandats pour le président. Toutefois, celle-ci semble assouplir cette règle en prévoyant des exceptions. En effet, la nouvelle rédaction de l'article 116 dispose qu'en cas de renouvellement des élections présidentielle et législatives provoqué par la GANT au cours du second mandat présidentiel, le président peut candidater à sa propre succession, et ainsi réaliser un troisième mandat. Toutefois, l'esprit de la règle peut être détourné au regard de la connivence entre la majorité de la GANT et le président. L'usage de cette disposition permettrait alors de reconduire le président pour un troisième mandat en cas d'assentiment de l'électorat qui est le dernier décideur ${ }^{131}$. Dans cette même logique, le renouvellement des mandats parlementaires et présidentiel, qui devrait être un outil d'arbitrage dans l'esprit de la Constitution de 1982 pour contrevenir à un éventuel blocage des institutions ${ }^{132}$, pourrait se politiser s'il est utilisé lors de périodes favorables à la confirmation de la majorité en place.

Le caractère partisan de l'élection présidentielle est également mis en exergue par les lois électorales. La révision législative de mars $2018^{133}$ démontre, entre autres choses, la volonté de renforcer le positionnement des partis politiques lors des élections de juin 2018. En effet, au regard du score très juste obtenu pour le «oui » lors du référendum du 16 avril 2017 et de la victoire du «non » dans des circonscriptions électorales majeures pour l'AKP, la logique de coalition s'est imposée au président et à son parti pour s'assurer le succès lors des prochaines élections. En s'alliant avec le MHP (droite nationaliste), le président espère pouvoir être réélu à la tête de l'État ${ }^{134}$ avec sa majorité personnelle. Toutefois, le parti allié étant divisé et en perte de vitesse ${ }^{135}$, et malgré les révisions successives ayant mis l'accent sur l'exercice du pouvoir démocratique du peuple sans pour autant revenir sur le barrage national de $10 \%$ confectionné par les mêmes autorités de tutelle dénoncées dans lesdites réformes ${ }^{136}$, il fallait être assuré que les partis alliés puissent entrer à la GANT. Ainsi, la loi surnommée par la presse «loi sur l'alliance électorale», prévoyant notamment des mesures sur l'organisation du scrutin, dispose en son article 20 que le barrage électoral national de $10 \%$ est calculé selon le total des résultats obtenus par les partis alliés. Il y a là une rupture d'égalité évidente, surtout pour les partis ne formant pas d'alliance et qui restent soumis au barrage national dans sa totalité. En revanche, la loi n'a pas apporté de modification à l'article 52 de la loi $n^{\circ} 298$ qui met en place une rupture d'égalité quant au temps de parole durant la période électorale entre les partis politiques au pouvoir, le principal parti d'opposition, les partis représentés à la GANT et ceux qui n'y sont pas ${ }^{137}$. L'applicabilité de ce dispositif à l'élection présidentielle dès $2014^{138}$ laissait déjà apparaître une logique partisane au stade de la campagne dans l'arsenal législatif malgré le

129. Türkiye Büyük Millet Meclisi (Grande Assemblée nationale de Turquie), D. 26, Y.Y. 2, S.S. 447, p. 12-13.

130. L'article 101 de la Constitution de 1982 prévoit depuis 2007 une limitation à deux mandats pour le président (avant cette date, il s'agissait d'un septennat non renouvelable).

131. À condition qu'une majorité des trois cinquièmes de la GANT vote en ce sens.

132. Dans la version initiale de la Constitution de 1982, le président détenait en vertu de l'article 116 un droit de dissolution conditionné à l'absence de la formation d'un gouvernement pendant quarante-cinq jours (soit qu'il n'ait pas été formé, soit qu'il n'ait pas obtenu le vote de confiance) à la suite du rejet du vote de la question de confiance ou du vote d'une motion de censure à l'initiative des parlementaires ou du gouvernement. Le président avait la possibilité d'exercer ce pouvoir après avis du président de la GANT.

133. Loi no 7102 du 13 mars 2018 (Resmî Gazete, 16 mars 2018, 30362).

134. Il apparait cependant quelques controverses sur la comptabilisation des mandats du président actuel. En effet, Recep Tayyip Erdoğan a été élu à la présidence en 2014 sous le régime de la Constitution telle que révisée en 2007. Or, certains propos de l'entourage du président, formulés au conditionnel, laissent à penser que, s'il est réélu en 2019 sous l'égide de la Constitution révisée en 2017, la comptabilisation des mandats devrait recommencer. En cas de réélection en 2019 puis en 2024 celui-ci pourrait être président jusqu'en 2029.

135. Le MHP a obtenu aux dernières élections législatives environ $12 \%$ des voix lui apportant quarante sièges à la GANT, soit le plus petit effectif parmi les partis ayant réussi à surmonter le barrage électoral (en ligne: http://www.ysk.gov.tr/tr/1-kasim-2015--26-donem-milletvekili-genel-secimi/3413). 136. Dénoncé par exemple par Murat Sevinç (Türkiye’nin Anayasa imtihanı..., p. 102).

137. Voir É. Sales, D. Yılmaz, «L'élection du président de la République au suffrage universel direct en Turquie », Revue du droit public, ${ }^{\circ}{ }^{5}$, 2012 , p. 1454.

138. La loi sur l'élection présidentielle $n^{\circ} 6271$ du 19 janvier 2012 (Resmî Gazete, 26 janvier 2012, 28185) renvoie dans son article 13 relatif à la "propagande» durant la campagne à la loi no 298 pour les questions concernant la limitation du temps de parole. 
maintien d'une conception neutre de la présidence dans l'esprit, alors que la lettre n'imposait cette neutralité qu'à partir de l'élection.

En dernier lieu, le régime politique qui fait du chef de l'État à la fois une autorité de contrôle ${ }^{139}$ et une autorité gouvernante rend impossible l'élection d'une personnalité non partisane. Le parti politique présidentiel, et par voie de conséquence, plausiblement majoritaire, devient le centre de la gouvernance étatique, et donc de la prise de décision politique. La seule issue pour influer sur celle-ci en dehors des échéances électorales serait le développement des processus de la démocratie intra-partisane.

\section{Le développement de la démocratie intra-partisane: un correctif envisageable?}

L'évolution du statut de la présidence de la République et sa domination du régime politique par le biais de sa stature partisane pousse à rechercher des contre-pouvoirs, non plus dans les institutions représentatives elles-mêmes, mais au sein des rouages du parti politique majoritaire. L'organisation des partis politiques en Turquie fait appel à un constat: une organisation oligarchique et hiérarchisée $e^{140}$ dans laquelle les décisions viennent du haut et s'imposent à la base. La discipline partisane y est ainsi particulièrement développée, permettant aux dirigeants d'étendre leur autorité sur l'ensemble de l'appareil partisan. Ainsi, un parti majoritaire dirigé par un président titulaire de l'ensemble de l'exécutif ne laisse que peu de place à l'installation d'une forme de séparation des pouvoirs. À ce titre, la neutralité présidentielle reste encore le meilleur moyen, dans un régime connaissant des interactions aussi fortes entre le législatif et l'exécutif, de préserver une forme d'équilibre des pouvoirs. Mais, dans le cas d'une présidence partisane, un correctif à l'hégémonie présidentielle pourrait être apporté par le développement de la démocratie intra-partisane. Celle-ci, bien que ne suffisant pas à équilibrer le régime tel qu'il est conçu depuis la révision de 2017, pourrait mettre en place un terrain de dialogue, de contrôle et de renouvellement de la classe politique qui semble faire défaut à la nouvelle ingénierie constitutionnelle. Il apparaît, en dépit du déséquilibre entre le législatif et l'exécutif, que le moyen d'instaurer un contre-pouvoir efficace est d'assouplir la discipline partisane et de permettre une expression plus directe de la base du parti, notamment dans la majorité présidentielle, qui est la condition du développement de la démocratie intra-partisane. Il y a deux facteurs qui influent sur le développement de la démocratie intra-partisane: l'encadrement juridique des partis politiques (et notamment via la Constitution, la loi sur les partis politiques et le règlement des partis ainsi que la jurisprudence de la Cour constitutionnelle), mais aussi un facteur relatif à la culture politique du pays ${ }^{141}$.

D'un point de vue juridique, l'article 69 , alinéa $1^{\text {er }}$ de la Constitution de 1982 impose aux partis politiques le respect des principes démocratiques dans leurs activités, leur organisation interne et leur fonctionnement. En plus de faire place dès 1961 aux partis politiques dans sa Constitution, la Turquie a été l'un des premiers États à encadrer les partis politiques par une loi spécifique en $1965^{142}$, ce qui démontrait déjà une volonté de contrôle étatique sur ces organisations du fait d'une méfiance affirmée ${ }^{143}$. Alors que l'article 4, alinéa 2 de la loi sur les partis politiques de 1983 met en application l'article 69 de la Constitution ${ }^{144}$, l'article 93 de cette même loi dispose que:

Les travaux dans le parti, l'administration du parti, son contrôle, les élections des organes au sein du parti et les décisions prises par la présidence du parti, les organes du siège central et les groupes du parti, les actes et les opérations réalisés ne peuvent être en contradiction avec les règles du règlement du parti, du principe d'égalité entre les adhérents du parti, de la démocratie.

Cette même loi va jusqu'à préciser les différents échelons de l'organisation partisane dans son article 7 mais la procédure d'élection des membres les composant est laissée au libre choix du parti ${ }^{145}$. Et c'est précisément autour de la désignation, d'une part, des candidats à la présidence, et d'autre part, des candidats aux législatives, que se situe le principal enjeu consistant soit à développer des processus de démocratie intra-partisane, soit à accroître la discipline partisane en renforçant la figure du président partisan et chef de majorité.

Concernant la désignation des candidats aux législatives, le statut partisan du chef de l'État lui offre la possibilité de nommer les candidats aux législatives qui l'accompagneront dans la campagne électorale

139. L'article 104 maintient dans son deuxième alinéa cette fonction du chef de l'État: «À ce titre, il représente l'unité de la République de Turquie et de la nation turque; il veille à l'application de la Constitution et au fonctionnement ordonné et harmonieux des organes de l'État ».

140. C'est ce que rapporte Murat Yanık dans son analyse sur la démocratie intra-partisane en Turquie: «Alors que quand nous regardons les partis politiques de notre pays du point de vue de la démocratie intra-partisane, nous voyons, de manière hiérarchique, l'indiscutable souveraineté du leader du parti et de son cercle rapproché. Cette construction hiérarchique constitue un frein à la réalisation de la démocratie intra-partisane» (M. Yanık, Liberal Perspektif Rapor, p. 14).

141. Voir en ce sens A. F. Gökçe, «Siyasi partilerde parti içi demokrasi ve disiplin algısı: Türkiye», Akademik Araştırmalar ve Çalışmalar Dergisi (Journal of Academic Researches and Studies), $5^{\mathrm{e}}$ année, n⿳0 9, novembre 2013, p. 66; T. S. Küçük, Parti içi demokrasi, Istanbul, On iki levha Yayıncilik, 2015, p. 367 (issu d'une thèse soutenue en 2014 à l'université de Yeditepe).

142. Avec les pays comme la Grèce, le Portugal, l’Espagne, à la suite de l’Italie (1947), et l'Allemagne (1949) (I. H. Șentürk, «Türk hukukunda siyasi partilerin sicileri tutulması", Uyuşmazlık Mahkemesi Dergisi, vol. 4, nº 4, janvier 2014, p. 465).

143. Voir S. Zariç, «Demokratikleşme ve etkin bir siyasal sistem oluşturma bağlamında Türkiye'de siyasi partilerde lider hegemonyası ve lider değişmi sorunsalı", Pamukkale Üniversitesi Sosyal Bilimler Enstitüsü Dergisi, n 8, 2011, p. 103.

144. «La fondation, le choix des organes, le fonctionnement, les activités et les décisions des partis politiques ne doivent pas être en contradiction avec les règles démocratiques dont les caractéristiques ont été déterminées par la Constitution» (Resmî Gazete, 24 avril 1983, p. 1).

145. Article 13 de la loi $n^{\circ} 2820$ sur les partis politiques (Resmî Gazete, 24 avril 1983, 18027, p. 4). 
pour la double élection parlementaire et présidentielle. Cela conditionne les élus parlementaires à une forme de redevabilité envers le chef de parti qui se traduit par une loyauté envers le président de la République, empêchant ainsi tout contrôle sur l'action du présidentgouvernant. Il est donc nécessaire d'assouplir les liens entre les candidats aux législatives du parti et le candidat à la présidentielle en amont, lors de la désignation de ceux-ci afin d'inhiber toute loyauté systématique. La version initiale de la loi sur les partis politiques prévoyait, en son article 37 , l'obligation de mettre en place des primaires ${ }^{146}$ pour désigner les candidats du parti aux élections législatives ${ }^{147}$. Pourtant, cet article n'a jamais été appliqué, d'abord parce que le sixième article transitoire de la loi prévoyait l'interdiction des primaires pour les premières élections suivant le vote de la loi ${ }^{148}$, puis en raison de la révision de la loi sur les partis politiques en 1986 délaissant au parti le libre choix dans la procédure de désignation des candidats sous condition du respect de certains principes ${ }^{149}$. Les règlements des principaux partis tentent de faire bonne figure à cet égard. Le règlement de l'AKP, tout en donnant la compétence de principe au conseil d'administration centrale du parti (MKYK, cinquante membres), prévoit dans l'article 124, alinéa 3 de son règlement qu'il sera veillé à ce que les candidats d'au moins $50 \%$ des circonscriptions électorales soient désignés par le biais de primaires ou d'élections internes ${ }^{150}$, mais, selon T. S. Küçük, cette disposition n'a pas de valeur contraignante pour le conseil ${ }^{151}$. Il en va de même pour le MHP qui, à l'article 89 de son règlement, donne toute compétence en la matière au conseil d'administration centrale (soixante-quinze membres) ${ }^{152}$, alors que le HDP donne cette compétence à l'assemblée du parti (cent membres) ${ }^{153}$. Le CHP prévoit, quant à lui, dans son nouveau règlement de mars 2018, que la désignation par voie de primaires sera la priorité ${ }^{154}$. Mais en excluant certains cas de figure, comme par exemple les circonscriptions dans lesquelles le parti a obtenu moins de $10 \%$ des suffrages aux dernières élections, le règlement limite considérablement le nombre de primaires, et attribue une grande partie de cette compétence à l'assemblée du parti (soixante membres). Dans les faits, selon le rapport de Murat Yanık, lors des élections législatives de juin et novembre 2015 et exception faite d'une partie des candidats du CHP, tous les candidats des partis ont été investis par leur leader ${ }^{155}$. C'est ainsi que, concernant les élections législatives, la désignation des candidats par la base, c'est-à-dire par les adhérents du parti, reste tout à fait exceptionnelle, favorisant ainsi la discipline partisane à l'égard du leader. Sans une révision profonde de ce système par chaque parti, la confusion des pouvoirs législatif et exécutif mise en œuvre par la logique constitutionnelle risque d'être accentuée par le système de parti ainsi conçu.

La désignation des dirigeants est également une donnée importante puisqu'au regard des nouvelles dispositions mises en place, ce sont ces derniers qui seront les potentiels candidats aux élections présidentielles. L'article 15 de la loi sur les partis politiques impose la procédure à suivre en la matière en prévoyant une élection à la majorité absolue des voix du grand congrès par un vote à bulletin secret. Si la majorité n'est pas atteinte lors des deux premiers tours, c'est le candidat ayant obtenu le plus de voix qui doit être élu au troisième tour ${ }^{156}$. Toutefois, le mode de désignation des membres du grand congrès est laissé à la discrétion des partis politiques. La loi prévoit simplement la présence des membres de droit (les titulaires composant les autres organes centraux du parti ainsi que les députés et ministres du parti) et des membres élus, dont le nombre ne doit pas dépasser le double du nombre des députés composant la GANT ${ }^{157}$. Ainsi, la composition de cet organe n'est pas assez significative en termes d'effectif pour doter, non seulement le dirigeant du parti, mais surtout le potentiel «hyperprésident», d'une légitimité démocratique significative pour occuper la tête du parti, et potentiellement la tête de l'État.

La principale difficulté de l'organisation partisane provient du «système de délégués » qui permet aux hauts dirigeants de bénéficier d'une organisation verticalisée du parti et de pouvoir faire usage d'un contrôle hiérarchique sur leurs membres ${ }^{158}$. Ce système connaît par ailleurs quelques incohérences, la réélection récente de Kemal Kılıçdaroğlu à la tête du CHP a été beaucoup critiquée à ce titre $^{159}$. Mais il permet de garder, pour les hauts dirigeants

146. Il s'agirait ici de primaires fermées, c'est-à-dire réservées aux adhérents du parti.

147. Resmî Gazete, 24 avril 1983, 18027, p. 9.

148. Ibid., p. 26.

149. Article 9 de la loi no 3270 du 28 mars 1986 modifiant l’article 37 de la loi no 2820 sur les partis politiques (Resmî Gazete, 15 avril 1986, 19079, p. 3 ).

150. Règlement de l'AKP, article 124, alinéa 3, p. 74, en ligne: http://m.akparti.org.tr/site/akparti/parti-tuzugu.

151. T. S. Küçük, Parti içi demokrasi, p. 447.

152. Règlement du MHP, article 89, p. 125, en ligne: https://www.mhp.org.tr/usr_img/_mhp2007/kitaplar/mhp_parti_tuzugu_2009_opt.pdf.

153. Règlement du HDP, article 48, en ligne: http://www.hdp.org.tr/tr/parti/parti-tuzugu/1o.

154. Règlement du CHP, article 52, alinéa $1^{\text {er }}$, p. 55, en ligne: http://cdn.chp.org.tr/cms/o/Folder/CHP_Tuzuk_10_03_2018.pdf.

155. M. Yanık, Liberal Perspektif Rapor, p. 26.

156. Resmî Gazete, 24 avril 1983, 18027, p. 4.

157. Article 14 de la loi no 2820 sur les partis politiques (Resmî Gazete, 24 avril 1983, 18027, p. 4)

158. Voir A. F. Gökçe, «Siyasi partilerde parti...», p. 73.

159. L'éditorialiste du quotidien kémaliste Sözcü, Yilmaz Özdil, a ainsi souligné l'incohérence de la répartition des délégués dans les différentes provinces, celles ayant moins de suffrages pour le parti disposant proportionnellement de plus de délégués que les provinces votant en masse pour le CHP (Y. Özdil, «Kaybedenler kulübü», Sözcü, 4 février 2018, en ligne: https://www.sozcu.com.tr/2018/yazarlar/yilmaz-ozdil/kaybedenlerkulubu-2-2202794). 
du parti, un contrôle certain sur les délégués et le sens de leurs votes ${ }^{160}$. Avec un nombre peu élevé de délégués aux différents échelons, c'est finalement le suffrage indirect à quatre tours qui va permettre d'élire les hauts dirigeants du parti ${ }^{161}$. Les listes électorales des délégués sont réalisées par les dirigeants et les voix dissonantes peuvent être sanctionnées par le biais des outils disciplinaires utilisés inadéquatement par les partis ${ }^{162}$. Toutes ces pratiques constituent autant d'outils à la disposition du président partisan pour asseoir son emprise sur le parti politique tout en conservant les rênes de l'État.

Un autre élément accentuant l'aspect oligarchique de l'organisation partisane est le manque de renouvellement des leaders politiques. Les échecs électoraux n'ont par exemple aucune répercussion sur les têtes du parti qui parviennent à se maintenir. Le principal parti d'opposition n'a ainsi connu que huit personnes à la présidence du parti depuis Atatürk. Les règlements des partis sont assez révélateurs de ce constat. Celui de l'AKP prévoit qu'une même personne ne peut être le président du parti plus de quatre mandats sans interruption tout en excluant le(s) président(s) fondateur(s) du parti ${ }^{163}$. Le CHP, à l'instar du MHP, ne prévoit pas de limitation du mandat du président du parti. Il en va de même pour les coprésidents du HDP pour lesquels aucune limite n'est prévue. Ces règlements illustrent le fonctionnement oligarchique des partis et l'autorité des leaders dans la mesure où le dirigeant fondateur devient indéboulonnable ${ }^{164}$, ce qui diminue également les chances de voir une alternance politique, que ce soit à la tête du parti, mais surtout à la tête de l'État.

Il est essentiel que le mode de désignation des candidats aux législatives et des présidents de parti se démocratise, et surtout de permettre à la base du parti de garder un contrôle politique permanent sur leurs dirigeants. Cela pourrait potentiellement donner lieu à une césure entre le président partisan élu et son parti majoritaire à la GANT, et permettre d'avoir une forme de séparation des pouvoirs. Bien entendu, les deux organes pourraient dans ce cas avoir recours au renouvellement des élections, mais l'instauration d'un tel contrôle pourrait obliger un président hégémonique à maintenir un dialogue constant avec la base de son parti ${ }^{165}$. Enfin, il ne faut pas laisser s'installer une forme d'automaticité du lien entre le président de parti et le candidat du parti aux élections présidentielles. L'instauration d'un processus de nomination par les adhérents ou sympathisants du parti prenant la forme d'une primaire ouverte pourrait permettre de parvenir à des programmes politiques plus rassembleurs, assouplissant ainsi la polarisation que connaît actuellement le pays.

Le $1^{\text {er }}$ mai 2018.

160. Ce contrôle a été très poussé à l'AKP puisqu'il a abouti à la démission forcée de certains maires par les dirigeants du parti, et notamment le président Erdoğan. Le but de la manœuvre est essentiellement politique puisqu'elle vise à préparer les élections municipales (prévues en mars 2019) et législatives et présidentielle (prévues en novembre 2019), les municipalités concernées risquant d'échapper au parti présidentiel (M. Jégo, «En Turquie, Erdoğan fait le ménage dans son propre parti», Le Monde, 25 octobre 2017, p. 4).

161. Voir M. Yanik, Liberal Perspektif Rapor, p. 22.

162. Voir A. F. Gökçe, «Siyasi partilerde parti... », p. 72.

163. Règlement de l'AKP, article 75, p. 52.

164. Selon la thèse de doctorat de Murat Yanık, il y a une «identification» qui s'opère entre le leader et son parti (Parti içi demokrasi, thèse en droit public, université d'Istanbul, 2 avril 2002, 227 p., p. 170), alors que T. S. Küçük constate dans la sienne qu' 'il est quasiment impossible en Turquie de changer les leaders des partis. Les personnes qui sont présidents restent en principe toute leur vie en fonction sauf circonstances exceptionnelles comme le décès, la promotion au poste de la présidence de la République, le coup d'État militaire ou le scandale politique» (T. S. Küçük, Parti içi demokrasi, p. 421).

165. Ce qui suppose également que la composition du parti soit démocratisée, que l'expression de la base puisse plus facilement remonter dans les plus hautes instances du parti et que la désignation des membres de ces organes ne soit pas du seul ressort de la présidence de la République. 


\section{Annexes}

\section{Présentation succincte des partis politiques cités dans l'article}

AKP - Adalet ve Kalkınma Partisi (Parti de la justice et du développement): fondé en 2001, l'AKP est l'un des partis issus de la dissolution du FP. Il se place au centre-droit de l'échiquier politique et est généralement défini comme islamo-conservateur et libéral sur le plan économique. Le parti est présidé par l'actuel chef de l'État Recep Tayyip Erdoğan. Au pouvoir depuis 2002, l'AKP a pu composer seul sept gouvernements dirigés successivement par Abdullah Gül (2002-2003), Recep Tayyip Erdoğan (20032014), Ahmet Davutoğlu (2014-2016) et Binali Yıldırım (2016-2018). Abdullah Gül (2007-2014) et Recep Tayyip Erdoğan (depuis 2014) ont été deux présidents de la République ayant un ancrage dans ce parti.

ANAP - Anavatan Partisi (Parti de la mère patrie) : fondé en 1983, c'est un parti libéral de centre-droit présidé notamment par Turgut Özal jusqu'à son élection à la présidence de la République, mais qui garde un contrôle très étroit sur son parti durant son mandat. Le parti est à la tête de l'État de 1983 à 1991 avec quatre gouvernements majoritaires dirigés successivement par Turgut Özal (1983-1989), Yıldırım Akbulut (1989-1991) et Mesut Yilmaz (1991), dont une partie sous la présidence de son leader Özal (1989-1993). À la suite du décès de son leader, le parti a participé à quelques gouvernements de coalition dirigés par Mesut Yilmaz (en 1996 et de 1997 à 1999), puis a intégré le gouvernement de coalition dirigé par Bülent Ecevit (DSP) de 1999 à 2002.

CHP - Cumhuriyet Halk Partisi (Parti républicain du peuple) : fondé en 1923 par M. Kemal Atatürk, le parti est dissous de 1981 à 1992. C'est le parti héritier de l'idéologie kémaliste, placé au centre-gauche de l'échiquier politique. Il a participé au $52^{\mathrm{e}}$ gouvernement dirigé par Tansu Çiller du DYP (du 30 octobre 1995 au 6 mars 1996). C'est le principal parti d'opposition à l'AKP depuis sa prise de pouvoir en 2002. Le CHP est présidé actuellement par Kemal Kılıçdaroğlu.

DSP - Demokratik Sol Parti (Parti démocratique de gauche) : parti fondé en 1985 par Rahşan Ecevit, épouse de Bülent Ecevit (ancien leader du CHP de 1972 à 1980), alors privé de ses droits civiques, il reprend officiellement la tête du parti à partir de 1987. Le DSP, à l'instar du SHP, est alors placé sur le créneau politique du CHP dissous depuis 1981. Au centre-gauche de l'échiquier politique, il porte les valeurs de la social-démocratie. Le DSP participe à un gouvernement de coalition dirigé par Mesut Yllmaz comprenant donc l'ANAP et le DTP (Demokrat Türkiye Partisi - Parti démocrate de Turquie -, frontistes du DYP) et dénommé la coalition ANASOL-D. Puis, à partir de 1999, Bülent Ecevit dirige successivement deux gouvernements, le premier, composé uniquement de son parti, tient quelques mois, avant d'être remplacé de 1999 jusqu'à 2002 par un gouvernement de coalition comprenant l'ANAP et le MHP. Il n'a pas été question pour Bülent Ecevit, contrairement aux autres leaders politiques actifs depuis la II ${ }^{e}$ République, d'être candidat à la présidence de la République, celui-ci ne remplissant pas la condition d'éligibilité relative à la possession d'un diplôme de l'enseignement supérieur.

DYP - Doğru Yol Partisi (Parti de la juste voie): parti issu du Parti démocrate (Demokrat Parti - DP -, premier véritable parti d'opposition marquant le début du multipartisme en 1946) et du Parti de la justice (Adalet Partisi - AP -, fondé par Süleyman Demirel à la suite de la dissolution du DP par le coup d'État de 1960, et luimême dissous par le coup d'État de 1980). Fondé en 1983 à l'instigation de Süleyman Demirel, il se situe au centredroit de l'échiquier politique en défendant le libéralisme économique et le conservatisme politique. C'est Tansu Çiller qui prend la tête du parti à partir de la présidence du leader du parti Süleyman Demirel (1993-1999). Le DYP constitue l'une des principales forces politiques du pays de 1991 à 1997 avec des gouvernements de coalition composés du parti de gauche (le SHP à deux reprises puis le CHP). Après une tentative avec l'ANAP en 1996, le parti forme un gouvernement de coalition avec le Parti du bien-être (Refah Partisi - RP) de Necmettin Erbakan (le REFAHYOL).

FP - Fazilet Partisi (Parti de la vertu): fondé en 1997 en prévision de la dissolution du Parti du bien-être (Refah Partisi - RP) en 1998. Il s'agit d'un parti islamo-conservateur. La Cour constitutionnelle ordonne sa dissolution en 2001, donnant lieu à une scission politique de laquelle sont issus deux partis politiques toujours en activité: le Parti de la félicité (Saadet Partisi - SP -, allié avec le CHP pour les élections de juin 2018) et l'AKP.

HDP - Halkların Demokratik Partisi (Parti démocratique des peuples): fondé en 2012, il est issu d'une lignée de partis politiques "pro-kurdes» (Halkın Emek Partisi, Demokrasi Partisi, Halkın Demokrasi Partisi, Barış ve Demokrasi Partisi, etc.) ayant généralement fait l'objet d'une dissolution. Situé à gauche de l'échiquier politique, le parti se présente comme progressiste. Disposant d'un groupe parlementaire à la GANT depuis 2007, il en constitue la troisième force politique à l'issue des législatives de 2015. Cependant, le HDP a été touché par les purges massives effectuées à la suite de la tentative de coup d'État de juillet 2016. Initialement coprésidé par Selahattin Demirtaş et Figen Yüksekdağ, le parti est actuellement dirigé par Pervin Buldan et Sezai Temelli.

İP - İyi Parti (Le bon parti): fondé en octobre 2017 par Meral Akşsener, il est composé notamment par les dissidents du MHP refusant l'alliance avec l'AKP. Le noyau du parti se situe logiquement dans la lignée idéologique du MHP - soit le nationalisme - mais il souhaite effectuer une synthèse des différentes idéologies dominantes afin de toucher un large électorat, notamment les laïcs du CHP, et vise ainsi à se rapprocher du centre-droit de l'échiquier politique. 
MHP - Milliyetçi Hareket Partisi (Parti de l'action nationaliste): fondé en 1969 sous le leadership d'Alparslan Türkeş, il s'agit d'un parti nationaliste situé à l'extrême droite de l'échiquier politique. Dirigé par Devlet Bahçeli depuis 1997, le MHP a participé au gouvernement de coalition mené par Bülent Ecevit entre 1999 et 2002 (avec l'ANAP) en étant le deuxième parti politique du pays à la suite des élections législatives de 1999. Actuellement, le MHP forme une alliance politique avec l'AKP, débutée dans le cadre du référendum sur la révision constitutionnelle de 2017 et qui se poursuit pour les élections législatives et présidentielle de juin 2018.

SHP - Sosyal Demokrat Halkçı Parti (Parti social-démocrate populaire) : fondé en 1985 par l'union du HP (Halkçı Parti - Parti populiste) et du SODEP (Sosyal Demokrasi Partisi - Parti de la social-démocratie), il se place logiquement dans la lignée du CHP. Le SHP est présidé par Erdal Inönü (fils de l’ancien président İsmet Inönü - 1938-1950). Idéologiquement placé au centre-gauche de l'échiquier politique, le parti porte les valeurs de la social-démocratie et celles du kémalisme, dont notamment la laïcité. Le SHP, en tant que troisième force politique à la GANT, forme un gouvernement de coalition avec le DYP de Süleyman Demirel et dirigé par celui-ci de 1991 à 1993. Le parti soutient la candidature de Demirel à la présidence de la République, puis forme un second gouvernement de coalition avec le DYP dirigé par Tansu Çiller de 1993 à 1995. Le SHP fusionne avec le CHP à partir de 1995.

\section{Principaux repères chronologiques}

1980: Coup d'État du 12 septembre mené par le général Kenan Evren.

1981: Dissolution des partis politiques et privation des droits civiques des principaux dirigeants de la II ${ }^{e}$ République.

1983: Autorisation de créer des partis politiques mais avec l'accord du Conseil de sécurité nationale. Trois partis sont ainsi autorisés à se présenter aux élections législatives de 1983: l'ANAP, le MDP (Milliyetçi Demokrasi Partisi - Parti de la démocratie nationaliste) et le HP (Halkçı parti - Parti populiste). Victoire de l'ANAP qui s'installe au pouvoir jusqu'en 1991.

1987: Organisation du référendum visant à lever l'interdiction dont sont frappés les anciens dirigeants politiques. Malgré une campagne pour le «non» de l'ANAP alors majoritaire, le « oui » l'emporte de justesse. Les dirigeants politiquement déchus sont alors autorisés à créer des nouvelles formations politiques, les anciennes demeurant interdites jusqu'en 1992.

1991 : Première alternance politique sous la III ${ }^{e}$ République marquant une période de gouvernements de coalition s'étendant jusqu'en 2002 et considérée parfois comme une période d'instabilité politique.

1997 : Mémorandum de l'armée du 27 février. Necmettin Erbakan du Parti du bien-être (Refah Partisi - RP -, islamo-conservateur), alors Premier ministre, est écarté du pouvoir.

2002: Élections législatives anticipées marquant la victoire de l'AKP et le début de sa domination politique.

2007: Crise de l'élection présidentielle d'Abdullah Gül avec une tentative d'interférence de l'armée et de la Cour constitutionnelle débouchant sur la réforme de l'élection présidentielle au suffrage universel direct.

2013: Mouvement de contestation populaire du Gezi.

2014: Première élection présidentielle au suffrage universel direct voyant la victoire du Premier ministre Recep Tayyip Erdoğan dès le premier tour.

2016: Tentative de coup d'État du 15 juillet. Mise en œuvre de l'état d'urgence, reconduit systématiquement depuis. Purges massives dans la fonction publique, la magistrature, chez les universitaires et dans l'armée.

2017: Référendum portant sur l'adoption de la loi de révision constitutionnelle parachevant le présidentialisme amorcé par la pratique du régime politique.

2018 : Premières élections législatives et présidentielle simultanées, marquant l'entrée en vigueur de la révision constitutionnelle de 2017 dans son intégralité. 